Louisiana State University

LSU Digital Commons

Faculty Publications

Department of Chemistry

9-15-2013

\title{
Spectroscopic, computational modeling and cytotoxicity of a series of meso-phenyl and meso-thienyl-BODIPYs
}

\author{
Jaime H. Gibbs \\ Louisiana State University \\ Larry T. Robins \\ Louisiana State University \\ Zehua Zhou \\ Louisiana State University \\ Petia Bobadova-Parvanova \\ Rockhurst University \\ Michael Cottam \\ Rockhurst University
}

See next page for additional authors

Follow this and additional works at: https://digitalcommons.Isu.edu/chemistry_pubs

\section{Recommended Citation}

Gibbs, J., Robins, L., Zhou, Z., Bobadova-Parvanova, P., Cottam, M., McCandless, G., Fronczek, F., \& Vicente, M. (2013). Spectroscopic, computational modeling and cytotoxicity of a series of meso-phenyl and mesothienyl-BODIPYs. Bioorganic and Medicinal Chemistry, 21 (18), 5770-5781. https://doi.org/10.1016/ j.bmc.2013.07.017

This Article is brought to you for free and open access by the Department of Chemistry at LSU Digital Commons. It has been accepted for inclusion in Faculty Publications by an authorized administrator of LSU Digital Commons. For more information, please contact ir@lsu.edu. 


\section{Authors}

Jaime H. Gibbs, Larry T. Robins, Zehua Zhou, Petia Bobadova-Parvanova, Michael Cottam, Gregory T. McCandless, Frank R. Fronczek, and M. Graça H. Vicente 


\title{
Spectroscopic, computational modeling and cytotoxicity of a series of meso-phenyl and meso-thienyl-BODIPYs
}

\author{
Jaime H. Gibbs ${ }^{a}$, Larry T. Robins ${ }^{a}$, Zehua Zhou ${ }^{a}$, Petia Bobadova-Parvanova ${ }^{b}$, Michael \\ Cottam $^{b}$, Gregory T. McCandless ${ }^{a}$, Frank R. Fronczek ${ }^{a}$, and M. Graça H. Vicente ${ }^{a,{ }^{*}}$ \\ aLouisiana State University, Department of Chemistry, Baton Rouge, LA 70803, USA

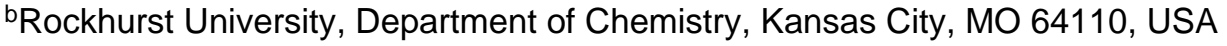

\begin{abstract}
A series of twenty-two BODIPY compounds were synthesized, containing various meso-phenyl and meso-thienyl groups, and their spectroscopic and structural properties were investigated using both experimental and computational methods. Further functionalization of the BODIPY framework via iodination at the 2,6-pyrrolic positions was explored in order to determine the effect of these heavy atoms on the photophysical and cytotoxicity of the meso-aryl-BODIPYs. BODIPYs bearing meso-thienyl substituents showed the largest red-shifted absorptions and emissions and reduced fluorescence quantum yields. The phototoxicity of the BODIPYs in human carcinoma HEp2 cells depends on both the presence of iodines and the nature of the meso-aryl groups. Six of the eleven 2,6-diiodo-BODIPYs investigated showed at least a sevenfold enhancement in phototoxicity $\left(\mathrm{IC}_{50}=3.5-28 \mu \mathrm{M}\right.$ at $\left.1.5 \mathrm{~J} / \mathrm{cm}^{2}\right)$ compared with the non-iodinated BODIPYs, while the others showed no cytotoxicity, while their singlet oxygen quantum yields ranged from 0.02 to 0.76 . Among the series investigated, BODIPYs 2a and 4a bearing electrondonating meso-dimethoxyphenyl substituents showed the highest phototoxicity and dark/ phototoxicity ratio, and are therefore the most promising for application in PDT.
\end{abstract}

\section{Keywords \\ BODIPY; PDT; Fluorescence; Phototoxicity; Cellular uptake}

\section{Introduction}

The high versatility of BODIPY (or 4,4-difluoro-4-bora-3a,4a-diaza-s-indacene) dyes has led to their investigation as fluorophores in a variety of applications, including biological labelling and imaging, metal ion sensing and as $\mathrm{pH}$ indicators. ${ }^{1-4}$ Recently BODIPYs have been proposed for application as photosensitizers in the photodynamic therapy (PDT) of cancers. ${ }^{5,6}$

(C) 2013 Elsevier Ltd. All rights reserved.

*Corresponding author. Tel.: +1 225578 7405; fax: +1 225578 3458. vicente@1su.edu (M.G.H. Vicente).

Supplementary data

Supplementary data (absorption and emission Figures for all $\beta$-free BODIPYs and absorption Figures for all di-iodo-BODIPYs in dichloromethane and $\mathrm{THF},{ }^{1} \mathrm{H}$ and ${ }^{13} \mathrm{C}$ NMR, cytotoxicity plots and X-ray crystal structure reports) associated with this article can be found, in the online version, at http://dx.doi.org/10.1016/j.bmc.2013.07.017. 
PDT is a process that combines three components, a photosensitizer, light and oxygen, in such a way that energy is transferred from light to molecular oxygen to generate reactive oxygen species, including singlet oxygen, that are highly cytotoxic to tissues. ${ }^{7,8}$ Two porphyrin-based macrocycles are FDA-approved as photosensitizers and several other porphyrinoids are under investigation for the PDT treatment of various neoplastic and nonmalignant conditions in dermatology, ophthalmology and cardiology. ${ }^{9,10}$ Characteristics of 'ideal' photosensitizers include preferential accumulation in target tissue, a triplet state of adequate energy $\left(E_{\mathrm{T}} \geq 95 \mathrm{~kJ} \mathrm{~mol}^{-1}\right)$ for efficient energy transfer, high quantum yields of the triplet state $\left(\Phi_{\mathrm{T}}>0.4\right)$, long triplet state lifetimes $\left(\tau_{\mathrm{T}}=1 \mu \mathrm{s}\right)$, high photostability, high absorption coefficients at the therapeutic excitation window $(650-800 \mathrm{~nm})$, and low dark but high phototoxicity. ${ }^{7,8}$ BODIPY-based dyes can be synthesized with extended $\pi$-systems for excitation within the therapeutic window, however their high phototoxicity/low dark toxicity requirement and the structural features that optimize tumor cell uptake and cytotoxicity remain poorly understood.

The incorporation of heavy halogen atoms, such as bromine or iodine, onto the BODIPY platform generates useful precursors for cross-coupling and nucleophilic substitution reactions, ${ }^{11-13}$ and can also lead to effective photosensitizers for PDT applications. ${ }^{5,6}$ The addition of heavy atoms to the BODIPY core has the potential to cause enhanced intersystem crossing from the singlet to the triplet excited state that controls the production of singlet oxygen, the main cytotoxic species in PDT, due to spin-orbit coupling by the 'heavy atom effect' ${ }^{13-15}$ In particular, the introduction of iodine atoms at the 2- and/or 6positions of the BODIPY tends to favor intersystem crossing and singlet oxygen generation, while substitution at the 3,5-positions is reported to lead to fluorescence enhancement. ${ }^{13}$ Furthermore, the absorption and emission profiles of halogenated BODIPYs are also expected to be red-shifted compared with their non-halogenated analogs.

On the other hand, the use of isotopically and radioactively labeled heavy atoms, such as ${ }^{123} \mathrm{I},{ }^{124} \mathrm{I}$, and ${ }^{131} \mathrm{I}$, can allow iodinated BODIPYs to be utilized in various bioimaging applications. Radioactive isotopically labeled iodine has been effectively used in single photon emission computed tomography (SPECT) and positron emission tomography (PET) imaging studies. ${ }^{16,17}$ In previous work, ${ }^{123}$ I nuclei have been successfully used in nuclear medicine including blood flow, myocardial, and thyroid scintigraphy and for uptake measurements in tumors. ${ }^{18}$ The use of radioactively labeled iodine has gained popularity in bioimaging for its longer half-life (ca. $13 \mathrm{~h}$ ) compared with other commonly used radio agents, including fluorine. ${ }^{19}$ Incorporation of targeting moieties into the BODIPY platform is usually performed through post-synthetic modifications ${ }^{20-22}$ making radioiodine-labeled BODIPYs suitable for potential use in SPECT and PET modalities.

Herein we report the synthesis of a series of eleven photo-stable meso-aryl-BODIPYs from 2,4-dimethylpyrrole and various aryl aldehydes. Furthermore, iodination at the 2,6-positions gave the corresponding diiodo-BODIPY derivatives with high yield and selectivity. These 3,5-dimethyl substituted BODIPYs can undergo Knoevenagel condensation reactions with aldehydes to give mono- and di-styryl functionalized long wavelength absorbing BODIPY dyes, within the biological window suitable for PDT. ${ }^{23-25}$ In this study we investigated the 
effects of the meso-aryl substituents on the spectroscopic and cytotoxic properties of a series of eleven BODIPYs and their 2,6-diiodo derivatives.

\section{Experimental Section}

\subsection{Chemistry}

All reagents and solvents were purchased from either Sigma Aldrich or Alfa Aesar as reagent grade and used without further purification. Reactions were monitored by TLC using $0.2 \mathrm{~mm}$ silica with UV indicator (UV254). Column chromatography was performed using Sorbent Technologies $60 \AA$ A silica gel (230-400 mesh). All ${ }^{1} \mathrm{H}$ NMR and ${ }^{13} \mathrm{C}$ NMR spectra were obtained using a Bruker DPX-400, AV-400, or DPX-250 spectrometer (400 MHz or $250 \mathrm{MHz}$ for ${ }^{1} \mathrm{H}, 100 \mathrm{MHz}$ for ${ }^{13} \mathrm{C}$ ) in deuterated chloroformas solvent with trimethylsilane as an internal indicator. Chemical shifts $(\delta)$ are reported in ppm with $\mathrm{CDCl}_{3}\left({ }^{1} \mathrm{H}: 7.27\right.$ ppm; ${ }^{13} \mathrm{C}$ : 77.16) used as reference. Coupling constants $(J)$ are reported in Hertz (Hz). High resolution ESI and MALDI mass spectra were obtained using an Agilent Technologies 6210 ESI-TOF Mass Spectrometer or a Bruker UltrafleXtreme MALDI-TOF/TOF. Melting points were determined using a MEL-TEMP electrothermal instrument. 5'-Bromo-[2,2'bithiophen]-5-carbaldehyde was synthesized in $64 \%$ yield as previously reported. ${ }^{26}$

2.1.1. General procedure for synthesis of BODIPYs 1-11-In an oven dried flask, 2,4-dimethylpyrrole $(0.9990 \mathrm{~g}, 10.5 \mathrm{mmol})$ and the corresponding aryl aldehyde $(5.0 \mathrm{mmol})$ were dissolved in dry dichloromethane (DCM, $300 \mathrm{~mL}$ ). Boron trifluoride diethyl etherate $\left(\mathrm{BF}_{3} \cdot \mathrm{OEt}_{2}, 0.15 \mathrm{~mL}\right)$ was added drop-wise and the mixture was stirred at room temperature under nitrogen atmosphere for $48 \mathrm{~h}$ (until TLC revealed disappearance of the aldehyde). 2,3Dichloro-5,6-dicyano- $p$-benzoquinone (DDQ, $1.1578 \mathrm{~g}, 5.1 \mathrm{mmol})$ in DCM (5 mL) was added to the solution and stirred for $1 \mathrm{~h}$. Triethylamine $(3.8216 \mathrm{~g}, 37.5 \mathrm{mmol})$ was then added to the mixture and stirred for $30 \mathrm{~min}$ followed by the introduction of $\mathrm{BF}_{3} \cdot \mathrm{OEt}_{2}(6.17$ $\mathrm{mL}, 50 \mathrm{mmol})$ in $\mathrm{DCM}(10 \mathrm{~mL})$ and stirred for $3 \mathrm{~h}$. The mixture was poured into water and the organic layer was washed twice with saturated sodium chloride. The organic layer was passed through a bed of anhydrous $\mathrm{Na}_{2} \mathrm{SO}_{4}$ and the solvent was evaporated under reduced pressure. The resulting residue was passed through a silica plug using dichloromethane as eluent. The solvent was again removed under vacuum and the crude product was purified by silica gel flash chromatography using $30 \%$ dichloromethane in petroleum ether.

\subsubsection{BODIPY 1 (4,4-difluoro-8-phenyl-1,3,5,7-tetramethyl-4-bora-3a,4a-diaza-s-} indacene): Obtained as a red solid $(0.3529 \mathrm{~g})$ in $22 \%$ yield, mp: $168-169{ }^{\circ} \mathrm{C} ;{ }^{1} \mathrm{H}$ NMR (400 $\left.\mathrm{MHz}, \mathrm{CDCl}_{3}\right) \delta 7.49$ (dd, $J=5.2 \mathrm{~Hz}, 1.4,3 \mathrm{H}, o, m$-phenyl $\mathrm{H}$ ), 7.29 (dd, $J=7.2$ and $2.2 \mathrm{~Hz}$, $2 \mathrm{H}, p$-phenyl H), 5.98 (s, $2 \mathrm{H}, \beta$-pyrrole $\mathrm{H}$ ), $2.56\left(\mathrm{~s}, 6 \mathrm{H}, 3,5-\mathrm{CH}_{3}\right), 1.38$ (s, $6 \mathrm{H}, 1,7$ $\left.\mathrm{CH}_{3}\right) ;{ }^{13} \mathrm{C}$ NMR $\left(100 \mathrm{MHz}, \mathrm{CDCl}_{3}\right) \delta 155.83,143.56,142.14,135.40,131.84,129.53$, 129.34, 128.35, 121.60, 14.98, 14.73; HRMS (ESI-TOF) $\mathrm{m} / \mathrm{z} 325.1709[\mathrm{M}+\mathrm{H}]^{+}$, calculated for $\mathrm{C}_{19} \mathrm{H}_{20} \mathrm{BF}_{2} \mathrm{~N}_{2}: 325.1688$. The NMR data is in agreement with that previously reported. ${ }^{27}$

\subsubsection{BODIPY 2 (4,4-difluoro-8-(3,5-dimethoxyphenyl)-1,3,5,7-tetramethyl-4-}

bora-3a,4a-diaza-s-indacene): Obtained as a red-orange solid $(1.1098 \mathrm{~g})$ in $58 \%$ yield, $\mathrm{mp}$ $=162{ }^{\circ} \mathrm{C}$ (decomposes); ${ }^{1} \mathrm{H} \mathrm{NMR}\left(400 \mathrm{MHz}, \mathrm{CDCl}_{3}\right) \delta 6.54(\mathrm{t}, J=2.0 \mathrm{~Hz}, 2 \mathrm{H}, m$-phenyl $\mathrm{H}), 6.46$ (d, $J=2.0 \mathrm{~Hz}, 1 \mathrm{H}, p$-phenyl H), 5.99 (s, 2H, $\beta$-pyrrole H), 3.80 (s, 6H, $\mathrm{OCH}_{3}$ ), 2.56 
(s, 6H, 3,5- $\left.\mathrm{CH}_{3}\right), 1.55\left(\mathrm{~s}, 6 \mathrm{H}, 1,7-\mathrm{CH}_{3}\right) ;{ }^{13} \mathrm{C} \mathrm{NMR}\left(100 \mathrm{MHz}, \mathrm{CDCl}_{3}\right) \delta 161.59,155.46$, 143.10, 141.33, 136.57, 131.06, 121.08, 105.90, 100.92, 55.52, 14.54, 14.17; HRMS (ESITOF) $m / z 385.1894[\mathrm{M}+\mathrm{H}]^{+}$, calculated for $\mathrm{C}_{21} \mathrm{H}_{24} \mathrm{BF}_{2} \mathrm{~N}_{2} \mathrm{O}_{2}: 385.1899$. The NMR data is in agreement with that previously reported. ${ }^{28}$

\subsubsection{BODIPY 3 (4,4-difluoro-8-(3, 5-di-tert-butylphenyl)-1,3,5,7-tetramethyl-4-}

bora-3a,4a-diaza-s-indacene): Obtained as a red-orange solid $(0.5084 \mathrm{~g})$ in $23 \%$ yield, $\mathrm{mp}$ $=115{ }^{\circ} \mathrm{C}$ (decomposes); ${ }^{1} \mathrm{H}$ NMR $\left(400 \mathrm{MHz}, \mathrm{CDCl}_{3}\right) \delta 7.47(\mathrm{t}, J=1.8 \mathrm{~Hz}, 1 \mathrm{H}, p$-phenyl H), 7.13 (d, $J=1.3 \mathrm{~Hz}, 2 \mathrm{H}, o$-phenyl H), 5.98 (s, 2H, $\beta$-pyrrole $\mathrm{H}$ ), 2.56 (s, 6H, 3,5- $\mathrm{CH}_{3}$ ), 1.36 (s, $\left.6 \mathrm{H}, 1,7-\mathrm{CH}_{3}\right), 1.32$ (s, $18 \mathrm{H}, t$-butyl H); ${ }^{13} \mathrm{C} \mathrm{NMR}\left(100 \mathrm{MHz}, \mathrm{CDCl}_{3}\right) \delta 155.08,151.96$, 143.25, 143.17, 133.97, 131.52, 122.08, 121.82, 120.99, 35.08, 31.40, 14.56, 14.12; HRMS (ESI-TOF) $m / z$ 437.2977 [M+H] $]^{+}$, calculated for $\mathrm{C}_{27} \mathrm{H}_{36} \mathrm{BF}_{2} \mathrm{~N}_{2}: 437.2940$. The NMR data is in agreement with that previously reported. ${ }^{29}$

\subsubsection{BODIPY 4 (4,4-difluoro-8-(3,4-dimethoxyphenyl)-1,3,5,7-tetramethyl-4-}

bora-3a,4a-diaza-s-indacene): Obtained a bright red solid $(0.4756 \mathrm{~g})$ in $25 \%$ yield, $\mathrm{mp}=$ 182-183 ${ }^{\circ} \mathrm{C} .{ }^{1} \mathrm{H}$ NMR $\left(400 \mathrm{MHz}, \mathrm{CDCl}_{3}\right) \delta 6.97(\mathrm{~d}, J=7.8 \mathrm{~Hz}, 1 \mathrm{H}, 5$-phenyl H), 6.82 (dd, $J=8.7$ and $1.5 \mathrm{~Hz}, 2 \mathrm{H}, 6$-phenyl H), 6.78 (d, $J=1.5 \mathrm{~Hz}, 2$-phenyl H), 5.98 (s, 2H, $\beta$-pyrrole $\mathrm{H}), 3.94\left(\mathrm{~s}, 3 \mathrm{H}, \mathrm{OCH}_{3}\right), 3.84\left(\mathrm{~s}, 3 \mathrm{H}, \mathrm{OCH}_{3}\right), 2.54\left(\mathrm{~s}, 6 \mathrm{H}, 3,5-\mathrm{CH}_{3}\right), 1.47(\mathrm{~s}, 6 \mathrm{H}, 1,7-$ $\left.\mathrm{CH}_{3}\right) ;{ }^{13} \mathrm{C}$ NMR $\left(100 \mathrm{MHz}, \mathrm{CDCl}_{3}\right) \delta 155.38,149.79,149.50,143.16,141.58,131.72$, $127.13,121.11,120.42,111.52,111.07,56.11,55.91,14.57,14.45$; HRMS (ESI-TOF) $\mathrm{m} / \mathrm{z}$ $385.1893[\mathrm{M}+\mathrm{H}]^{+}$, calculated for $\mathrm{C}_{21} \mathrm{H}_{24} \mathrm{BF}_{2} \mathrm{~N}_{2} \mathrm{O}_{2}: 385.1899$. The NMR data is in agreement with that previously reported. ${ }^{30}$

2.1.1.5. BODIPY 5 (4,4-difluoro-8-(4-(methoxycarbonyl) phenyl)-1,3,5,7-tetramethyl-4bora-3a,4a-diaza-s-indacene): Obtained a bright red solid $(0.4367 \mathrm{~g})$ in $23 \%$ yield, $\mathrm{mp}=$ 181-182 ${ }^{\circ} \mathrm{C} ;{ }^{1} \mathrm{H}$ NMR $\left(400 \mathrm{MHz}, \mathrm{CDCl}_{3}\right) \delta 8.19(\mathrm{~d}, J=7.9 \mathrm{~Hz}, 2 \mathrm{H}, m$-phenyl H), $7.42(\mathrm{~d}, J$ $=7.9 \mathrm{~Hz}, 2 \mathrm{H}, o$-phenyl H), $5.99(\mathrm{~s}, 2 \mathrm{H}, \beta$-pyrrole $\mathrm{H}), 3.97\left(\mathrm{~s}, 3 \mathrm{H}, \mathrm{COOCH}_{3}\right), 2.56(\mathrm{~s}, 6 \mathrm{H}$, 3,5- $\left.\mathrm{CH}_{3}\right), 1.35\left(1,7-\mathrm{CH}_{3}\right) ;{ }^{13} \mathrm{CNMR}\left(100 \mathrm{MHz}, \mathrm{CDCl}_{3}\right) \delta 166.47,156.00,142.88,140.21$, 139.95, 130.80, 130.37, 128.39, 121.48, 52.40, 14.62, 14.51; HRMS (ESI-TOF) $\mathrm{m} / \mathrm{z}$ $383.1746[\mathrm{M}+\mathrm{H}]^{+}$, calculated for $\mathrm{C}_{21} \mathrm{H}_{22} \mathrm{BF}_{2} \mathrm{~N}_{2} \mathrm{O}_{2}: 383.1742$. The NMR data is in agreement with that previously reported. ${ }^{31}$

\subsubsection{BODIPY 6 (4,4-difluoro-8-(4-bromophenyl)-1,3,5,7-tetramethyl-4-bora-3a,4a-} diaza-s-indacene): Obtained as a red solid $(0.3760 \mathrm{~g})$ in $19 \%$ yield, $\mathrm{mp}=172-173{ }^{\circ} \mathrm{C} ;{ }^{1} \mathrm{H}$ NMR $\left(400 \mathrm{MHz}, \mathrm{CDCl}_{3}\right) \delta 7.65(\mathrm{~d}, J=8.2 \mathrm{~Hz}, 2 \mathrm{H}, m$-phenyl H), $7.18(\mathrm{~d}, J=8.2 \mathrm{~Hz}, 2 \mathrm{H}, o-$ phenyl H), 5.99 (s, $2 \mathrm{H}, \beta$-pyrrole $\mathrm{H}), 2.55\left(\mathrm{~s}, 6 \mathrm{H}, 3,5-\mathrm{CH}_{3}\right), 1.41\left(\mathrm{~s}, 6 \mathrm{H}, 1,7-\mathrm{CH}_{3}\right) ;{ }^{13} \mathrm{C}$ NMR $\left(100 \mathrm{MHz}, \mathrm{CDCl}_{3}\right) \delta 155.91,142.92,140.03,133.96,132.45,131.19,129.84123 .27$, $121.47,14.65,14.61$; HRMS (ESI-TOF) $\mathrm{m} / z 403.0780[\mathrm{M}+\mathrm{H}]^{+}$, calculated for $\mathrm{C}_{19} \mathrm{H}_{19} \mathrm{BBrF}_{2} \mathrm{~N}_{2}: 403.0793$. The NMR data is in agreement with that previously reported. ${ }^{32}$

2.1.1.7. BODIPY 7 (4,4-difluoro-8-(thiophen-2-yl)-1,3,5,7-tetramethyl-4-bora-3a,4adiaza-s-indacene): Obtained a red solid $(0.3298 \mathrm{~g})$ in $20 \%$ yield, $\mathrm{mp}=190-191{ }^{\circ} \mathrm{C} ;{ }^{1} \mathrm{H}$ $\operatorname{NMR}\left(400 \mathrm{MHz}, \mathrm{CDCl}_{3}\right) \delta 7.50(\mathrm{~d}, J=5.0 \mathrm{~Hz}, 1 \mathrm{H}, 5$-thienyl H), $7.14(\mathrm{t}, J=4.4 \mathrm{~Hz}, 1 \mathrm{H}, 4$ thienyl H), 6.99 (d, $J=3.2 \mathrm{~Hz}, 1 \mathrm{H}, 3$-thienyl H), 6.00 (s, 2H, $\beta$-pyrrole H), 2.55 (s, 6H, 3,5- 
$\left.\mathrm{CH}_{3}\right), 1.58\left(\mathrm{~s}, 6 \mathrm{H}, 1,7-\mathrm{CH}_{3}\right) ;{ }^{13} \mathrm{C} \mathrm{NMR}\left(100 \mathrm{MHz}, \mathrm{CDCl}_{3}\right) \delta 156.07,143.50,134.63$, 134.00, 132.40, 127.81, 127.61, 127.41, 121.50, 14.65, 13.55; HRMS (ESI-TOF) $\mathrm{m} / \mathrm{z}$ $331.1306[\mathrm{M}+\mathrm{H}]^{+}$, calculated for $\mathrm{C}_{17} \mathrm{H}_{18} \mathrm{BF}_{2} \mathrm{~N}_{2} \mathrm{~S}: 331.1252$. The NMR data is in agreement with that previously reported. ${ }^{33}$

\subsubsection{BODIPY 8 (4,4-difluoro-8-([2, 2'-bithiophen]-2-yl)-1,3,5,7-tetramethyl-4-}

bora-3a,4a-diaza-s-indacene): Obtained a dark red solid $(0.4180 \mathrm{~g})$ in $20 \%$ yield, $\mathrm{mp}=157$ ${ }^{\circ} \mathrm{C}$ (decomposes); ${ }^{1} \mathrm{H}$ NMR $\left(400 \mathrm{MHz}, \mathrm{CDCl}_{3}\right) \delta 7.28\left(\mathrm{~d}, J=3.5 \mathrm{~Hz}, 1 \mathrm{H}, 5^{\prime}\right.$-bithienyl H), 7.23 (d, $J=3.5 \mathrm{~Hz}, 1 \mathrm{H}, 4$-bithienyl H), 7.20 (d, $J=3.5 \mathrm{~Hz}, 1 \mathrm{H}, 3$-bithienyl H), 7.06 (dd, $J=$ 4.9 and $3.7 \mathrm{~Hz}, 1 \mathrm{H}, 3^{\prime}$-bithienyl H), 6.89 (d, $J=3.5 \mathrm{~Hz}, 1 \mathrm{H}, 4^{\prime}$-bithienyl), 6.02 (s, $2 \mathrm{H}, \beta$ pyrrole $\mathrm{H}), 2.56\left(\mathrm{~s}, 6 \mathrm{H}, 3,5-\mathrm{CH}_{3}\right), 1.75\left(\mathrm{~s}, 6 \mathrm{H}, 1,7-\mathrm{CH}_{3}\right) ;{ }^{13} \mathrm{C} \mathrm{NMR}\left(100 \mathrm{MHz}, \mathrm{CDCl}_{3}\right) \delta$ 156.28, 143.44, 133.14, 128.65, 127.98, 125.02, 124.33, 123.73, 121.58, 14.66, 13.95; HRMS (ESI-TOF) $m / z, 413.1116[\mathrm{M}+\mathrm{H}]^{+}$, calculated for $\mathrm{C}_{21} \mathrm{H}_{20} \mathrm{BF}_{2} \mathrm{~N}_{2} \mathrm{~S}: 413.1129$.

\subsubsection{BODIPY 9 (4,4-difluoro-8-(5-bromothiophen-2-yl)-1,3,5,7-tetramethyl-4-}

bora-3a,4a-diaza-s-indacene): Obtained a dark red solid $(0.3740 \mathrm{~g})$ in $18 \%$ yield, $\mathrm{mp}=$ 167-168 ${ }^{\circ} \mathrm{C} ;{ }^{1} \mathrm{H}$ NMR $\left(400 \mathrm{MHz}, \mathrm{CDCl}_{3}\right) \delta 7.10(\mathrm{~d}, J=3.5 \mathrm{~Hz}, 1 \mathrm{H}, 4$-thienyl-H), 6.77 (d, $J$ $=3.9 \mathrm{~Hz}, 1 \mathrm{H}, 3$-thienyl-H), 6.01 (s, 2H, $\beta$-pyrrole $\mathrm{H}), 2.54\left(\mathrm{~s}, 6 \mathrm{H}, 3,5-\mathrm{CH}_{3}\right), 1.69(\mathrm{~s}, 6 \mathrm{H}$, $\left.1,7-\mathrm{CH}_{3}\right) ;{ }^{13} \mathrm{C} \mathrm{NMR}\left(100 \mathrm{MHz}, \mathrm{CDCl}_{3}\right) \delta 156.57,143.34,136.32,132.11,130.36,128.43$, $121.75,113.83,14.65,13.87$; HRMS (ESI-TOF) $\mathrm{m} / z 409.0315[\mathrm{M}+\mathrm{H}]^{+}$, Calculated for $\mathrm{C}_{17} \mathrm{H}_{17} \mathrm{BBrF}_{2} \mathrm{~N}_{2} \mathrm{~S}: 409.0357$.

\subsubsection{BODIPY 10 (4,4-difluoro-8-(5'-bromo-[2,2'-bithiophen]-2-yl)-1,3,5,7-} tetramethyl-4-bora-3a,4a-diaza-s-indacene): Obtained as a dark red solid $(0.7439 \mathrm{~g})$ from $5^{\prime}$-bromo-[2,2'-bithiophen]-5-carbaldehyde ${ }^{19}(1.3658 \mathrm{~g})$ in $30 \%$ yield, $\mathrm{mp}=205{ }^{\circ} \mathrm{C}$ (decomposes); ${ }^{1} \mathrm{H}$ NMR (400 MHz, $\left.\mathrm{CDCl}_{3}\right) \delta 7.14$ (d, $J=3.5 \mathrm{~Hz}, 1 \mathrm{H}, 4,4^{\prime}$-bithienyl-H), 7.01 (d, $J=3.5 \mathrm{~Hz}, 1 \mathrm{H}, 4,4^{\prime}$-bithienyl H), 6.97 (d, $J=3.9 \mathrm{~Hz}, 1 \mathrm{H}, 3,3^{\prime}$-bithienyl-H), 6.89 (d, $J=3.9 \mathrm{~Hz}, 1 \mathrm{H}, 3,3^{\prime}$-bithienyl-H), 6.03 (s, 2H, $\beta$-pyrrole H), 2.56 (s, 6H, 3,5- $\mathrm{CH}_{3}$ ), 1.73 (s, $\left.6 \mathrm{H}, 1,7-\mathrm{CH}_{3}\right) ;{ }^{13} \mathrm{C} \mathrm{NMR}\left(100 \mathrm{MHz}, \mathrm{CDCl}_{3}\right) \delta 156.42,143.36,135.64,133.70,132.29$, 130.75, 128.76, 124.41, 123.96, 121.64, 111.80, 14.67, 13.93; HRMS (ESI-TOF, negative ion) $m / z 489.0089[\mathrm{M}+\mathrm{H}]^{+}$, calculated for $\mathrm{C}_{21} \mathrm{H}_{17} \mathrm{BBrF}_{2} \mathrm{~N}_{2} \mathrm{~S}_{2}$ : 489.0078 .

\subsubsection{BODIPY 11 (4,4-difluoro-8-pentafluorophenyl-1,3,5,7-tetramethyl-4-bora-3a,} 4a-diaza-s-indacene): Obtained as a red solid $(0.3820 \mathrm{~g})$ in $52 \%$ yield, $\mathrm{mp}=109{ }^{\circ} \mathrm{C}$ (decomposes); ${ }^{1} \mathrm{H}$ NMR (400 MHz, $\mathrm{CDCl}_{3}$ ) $\delta 6.06$ (s, 2H, $\beta$-pyrrole H), 2.57 (s, 6H, 3,5$\left.\mathrm{CH}_{3}\right), 1.62\left(\mathrm{~s}, 6 \mathrm{H}, 1,7-\mathrm{CH}_{3}\right) ;{ }^{13} \mathrm{C} \mathrm{NMR}\left(100 \mathrm{MHz}, \mathrm{CDCl}_{3}\right) \delta 157.78,145.26,142.77$, 141.50, 139.44, 137.10, 130.99, 122.73, 122.26, 14.76, 13.57; HRMS (ESI-TOF) $\mathrm{m} / \mathrm{z}$ $415.1337[\mathrm{M}+\mathrm{H}]^{+}$, calculated for $\mathrm{C}_{19} \mathrm{H}_{15} \mathrm{BF}_{7} \mathrm{~N}_{2}: 415.1216$. The NMR data is in agreement with that previously reported. ${ }^{34}$

2.1.2. General procedure for lodination of BODIPYs 1-11-Iodic acid (2 equiv) was dissolved in a minimal amount of water and added drop-wise to a solution of the BODIPY (1 equiv) and iodine (2.5 equiv) in a solution of 50:50 ethanol/DCM $(\sim 35 \mu \mathrm{M}$ solution). The resulting mixture was stirred at $60^{\circ} \mathrm{C}$ for $2 \mathrm{~h}$. After cooling, the solvent was 
evaporated under vacuum and the resulting residue was purified by silica gel column chromatography using 50:50 hexanes/DCM for elution.

2.1.2.1. BODIPY 1a (4,4-difluoro-8-phenyl-2,6-di-iodo-1,3,5,7-tetramethyl-4-bora-3a, 4a-diaza-s-indacene): Obtained as a red solid $(0.1618 \mathrm{~g})$ in $91 \%$ yield from $1(0.100 \mathrm{~g}$, $0.3085 \mathrm{mmol})$, iodic acid $(0.1085 \mathrm{~g}, 0.6169 \mathrm{mmol})$, iodine $(0.0979 \mathrm{~g}, 0.7713 \mathrm{mmol}), \mathrm{mp}=$ 204-206 ${ }^{\circ} \mathrm{C} ;{ }^{1} \mathrm{H}$ NMR $\left(400 \mathrm{MHz}, \mathrm{CDCl}_{3}\right) \delta 7.54(\mathrm{dd}, J=5.2 \mathrm{~Hz}, 1.4,3 \mathrm{H}, o, p$-phenyl H), $7.26\left(\mathrm{dd}, J=7.2\right.$ and $2.2 \mathrm{~Hz}, 2 \mathrm{H}, m$-phenyl H), 2.65 (s, 6H, 3,5- $\left.\mathrm{CH}_{3}\right), 1.39$ (s, 6H, 1,7$\left.\mathrm{CH}_{3}\right) ;{ }^{13} \mathrm{C} \mathrm{NMR}\left(100 \mathrm{MHz}, \mathrm{CDCl}_{3}\right) \delta 156.78,145.37,141.38,134.74,131.30,129.54$, 129.47, 127.79, 85.65, 16.95, 16.04; HRMS (ESI-TOF) $\mathrm{m} / z$ 575.9461 [M] $]^{+}$, calculated for $\mathrm{C}_{19} \mathrm{H}_{17} \mathrm{BF}_{2} \mathrm{I}_{2} \mathrm{~N}_{2}: 575.9543$. The NMR data is in agreement with that previously reported. ${ }^{35}$

\subsubsection{BODIPY 2a (4,4-difluoro-2,6-di-iodo-8-(3,5-dimethoxyphenyl)-1,3,5,7-} tetramethyl-4-bora-3a,4a-diaza-s-indacene): Obtained as a red solid $(0.0952 \mathrm{~g})$ in $57 \%$ yield from $2(0.100 \mathrm{~g}, 0.2603 \mathrm{mmol})$, iodic acid $(0.1145 \mathrm{~g}, 0.6508 \mathrm{mmol})$, iodine $(0.0826 \mathrm{~g}$, $0.6508 \mathrm{mmol}), \mathrm{mp}=192-193{ }^{\circ} \mathrm{C} ;{ }^{1} \mathrm{HNMR}\left(400 \mathrm{MHz}, \mathrm{CDCl}_{3}\right) \delta 6.59(\mathrm{t}, J=2.0 \mathrm{~Hz}, 2 \mathrm{H}, m-$ phenyl H),6.41(d, $J=2.0 \mathrm{~Hz}, 1 \mathrm{H}, p$-phenyl H), $3.81\left(\mathrm{~s}, 6 \mathrm{H}, \mathrm{OCH}_{3}\right), 2.65\left(\mathrm{~s}, 6 \mathrm{H}, 3,5-\mathrm{CH}_{3}\right)$, 1.57 (s, 6H, 1,7- $\left.\mathrm{CH}_{3}\right) ;{ }^{13} \mathrm{CNMR}\left(100 \mathrm{MHz}, \mathrm{CDCl}_{3}\right) \delta 161.94,156.84,149.46,145.42$, 136.31, 131.12, 105.77, 101.45, 85.58, 55.65, 16.88, 16.06; HRMS (ESI-TOF) $\mathrm{m} / \mathrm{z} 636.9810$ $[\mathrm{M}+\mathrm{H}]^{+}$, calculated for $\mathrm{C}_{21} \mathrm{H}_{21} \mathrm{BF}_{2} \mathrm{I}_{2} \mathrm{~N}_{2} \mathrm{O}_{2}: 636.9832$.

\subsubsection{BODIPY 3a (4,4-difluoro-8-(3,5-di-t-butylphenyl)-2,6-di-iodo-1,3,5,7-} tetramethyl-4-bora-3a,4a-diaza-s-indacene): Obtained as a red solid $(0.3658 \mathrm{~g})$ in $93 \%$ yield from $3(0.250 \mathrm{~g}, 0.5729 \mathrm{mmol})$, iodic acid $(0.2016 \mathrm{~g}, 1.1458 \mathrm{mmol})$ and iodine $(0.1818$ g, $1.4323 \mathrm{mmol}$ ); ${ }^{1} \mathrm{H}$ NMR (400 MHz, $\left.\mathrm{CDCl}_{3}\right) \delta 7.54$ (t, $J=1.8 \mathrm{~Hz}, 1 \mathrm{H}, p$-phenyl H), 7.08 $(\mathrm{d}, J=1.3 \mathrm{~Hz}, 2 \mathrm{H}, o$-phenyl $\mathrm{H}), 2.66\left(\mathrm{~s}, 6 \mathrm{H}, 3,5-\mathrm{CH}_{3}\right), 1.37\left(\mathrm{~s}, 6 \mathrm{H}, 1,7-\mathrm{CH}_{3}\right), 1.33(\mathrm{~s}, 18 \mathrm{H}, t$ butyl H); ${ }^{13} \mathrm{C}$ NMR $\left(100 \mathrm{MHz}, \mathrm{CDCl}_{3}\right) \delta 156.40,152.48,145.35,142.94,133.76,131.39$, 122.46, 121.87, 85.45, 35.15, 31.40, 16.69, 15.99; HRMS (ESI-TOF) $\mathrm{m} / \mathrm{z} 688.0730[\mathrm{M}]^{+}$, calculated for $\mathrm{C}_{27} \mathrm{H}_{33} \mathrm{BF}_{2} \mathrm{I}_{2} \mathrm{~N}_{2}: 688.0794$.

\subsubsection{BODIPY 4a (4,4-difluoro-8-(3,4-dimethoxyphenyl)-2,6-di-iodo-1,3,5,7-} tetramethyl-4-bora-3a, 4a-diaza-s-indacene): Obtained as a red solid $(0.3907 \mathrm{~g})$ in $94 \%$ yield from $4(0.250 \mathrm{~g}, 0.6507 \mathrm{mmol})$, iodic acid $(0.2289 \mathrm{~g}, 1.3013 \mathrm{mmol})$, iodine $(0.2065 \mathrm{~g}$, $1.6268 \mathrm{mmol}), \mathrm{mp}=215-217^{\circ} \mathrm{C} ;{ }^{1} \mathrm{H}$ NMR $\left(400 \mathrm{MHz}, \mathrm{CDCl}_{3}\right) \delta 7.02(\mathrm{~d}, J=7.8 \mathrm{~Hz}, 1 \mathrm{H}, 5-$ phenyl H), 6.82 (dd, $J=8.7 \mathrm{~Hz}, 1.5,2 \mathrm{H}, 6$-phenyl H), 6.74 (d, $J=1.5 \mathrm{~Hz}, 2$-phenyl H), 3.98 $\left(\mathrm{s}, 3 \mathrm{H}, \mathrm{OCH}_{3}\right), 3.87\left(\mathrm{~s}, 3 \mathrm{H}, \mathrm{OCH}_{3}\right), 2.65\left(\mathrm{~s}, 6 \mathrm{H}, 3,5-\mathrm{CH}_{3}\right), 1.49\left(\mathrm{~s}, 6 \mathrm{H}, 1,7-\mathrm{CH}_{3}\right) ;{ }^{13} \mathrm{C} \mathrm{NMR}$ $\left(100 \mathrm{MHz}, \mathrm{CDCl}_{3}\right) \delta 156.70,150.10,149.97,145.37,141.28,131.62,126.78,120.33$, 111.76, 111.81, 85.58, 56.18, 56.01, 17.07, 16.01; HRMS (ESI-TOF) $\mathrm{m} / \mathrm{z} 635.9840[\mathrm{M}]^{+}$, calculated for $\mathrm{C}_{21} \mathrm{H}_{21} \mathrm{BF}_{2} \mathrm{I}_{2} \mathrm{~N}_{2} \mathrm{O}_{2}: 635.9754$. The NMR data is in agreement with that previously reported. ${ }^{36}$

2.1.2.5. BODIPY 5a (4,4-difluoro-2,6-di-iodo-8-(4-(methoxycarbonyl) phenyl)-1,3,5,7tetramethyl-4-bora-3a,4a-diaza-s-indacene): Obtained as a red solid $(0.3570 \mathrm{~g})$ in $86 \%$ yield from $5(0.250 \mathrm{~g}, 0.6541 \mathrm{mmol})$, iodic acid $(0.2301 \mathrm{~g}, 1.3082 \mathrm{mmol})$, iodine $(0.2075 \mathrm{~g}$, $1.6353 \mathrm{mmol}), \mathrm{mp}=215^{\circ} \mathrm{C}$ (decomposes); ${ }^{1} \mathrm{H}$ NMR $\left(400 \mathrm{MHz}, \mathrm{CDCl}_{3}\right) \delta 8.23(\mathrm{~d}, J=7.9$ 
$\mathrm{Hz}, 2 \mathrm{H}, m$-phenyl H), 7.40 (d, J = 7.9 Hz, 2H, o-phenyl H), 3.99 (s, 3H, $\mathrm{COOCH}_{3}$ ), 2.65 (s, $\left.6 \mathrm{H}, 3,5-\mathrm{CH}_{3}\right), 1.37\left(1,7-\mathrm{CH}_{3}\right) ;{ }^{13} \mathrm{C} \mathrm{NMR}\left(100 \mathrm{MHz}, \mathrm{CDCl}_{3}\right) \delta 166.24,157.34,145.07$, 139.81, 139.46, 131.34, 130.81, 130.66, 128.24, 86.04, 52.53, 17.14, 16.12; HRMS (ESITOF) $m / z 634.9663[\mathrm{M}+\mathrm{H}]^{+}$, calculated for $\mathrm{C}_{21} \mathrm{H}_{20} \mathrm{BF}_{2} \mathrm{I}_{2} \mathrm{~N}_{2} \mathrm{O}_{2}:$ 634.9676. The NMR data is in agreement with that previously reported. ${ }^{37}$

2.1.2.6. BODIPY 6a (4,4-difluoro-8-(4-bromophenyl)-2,6-di-iodo-1,3,5,7-tetramethyl-4bora-3a,4a-diaza-s-indacene): Obtained as a red solid $(0.4422 \mathrm{~g})$ in $71 \%$ yield from 6 $(0.250 \mathrm{~g}, 0.6202 \mathrm{mmol})$, iodic acid $(0.2182 \mathrm{~g}, 1.2405 \mathrm{mmol})$, iodine $(0.1968 \mathrm{~g}, 1.5505$ $\mathrm{mmol}), \mathrm{mp}=232{ }^{\circ} \mathrm{C}$ (decomposes); ${ }^{1} \mathrm{H} \mathrm{NMR}\left(400 \mathrm{MHz}, \mathrm{CDCl}_{3}\right) \delta 7.71(\mathrm{~d}, J=8.2 \mathrm{~Hz}, 2 \mathrm{H}$, $m$-phenyl H), 7.18 (d, $J=8.2 \mathrm{~Hz}, 2 \mathrm{H}, o$-phenyl H), $2.65\left(\mathrm{~s}, 6 \mathrm{H}, 3,5-\mathrm{CH}_{3}\right), 1.44$ (s, 6H, 1,7$\left.\mathrm{CH}_{3}\right) ;{ }^{13} \mathrm{C}$ NMR $\left(100 \mathrm{MHz}, \mathrm{CDCl}_{3}\right) \delta 157.27,145.13,139.64,133.69,132.80,131.08$, 129.66 123.91, 85.99, 17.28, 16.09; HRMS (ESI-TOF) $\mathrm{m} / \mathrm{z} 653.8638[\mathrm{M}]^{+}$, calculated for $\mathrm{C}_{19} \mathrm{H}_{16} \mathrm{BBrF}_{2} \mathrm{I}_{2} \mathrm{~N}_{2}: 653.8648$.

\subsubsection{BODIPY 7a (4,4-difluoro-2,6-di-iodo-8-(thiophen-2-yl)-1,3,5,7-tetramethyl-4-} bora-3a,4a-diaza-s-indacene): Obtained as a red solid $(0.5997 \mathrm{~g})$ in $79 \%$ yield from 7 $(0.250 \mathrm{~g}, 0.7571 \mathrm{mmol})$, iodic acid $(0.2664 \mathrm{~g}, 1.5142 \mathrm{mmol})$, iodine $(0.2402 \mathrm{~g}, 1.8928$ $\mathrm{mmol}), \mathrm{mp}=186-187^{\circ} \mathrm{C} ;{ }^{1} \mathrm{H} \mathrm{NMR}\left(400 \mathrm{MHz}, \mathrm{CDCl}_{3}\right) \delta 7.57(\mathrm{~d}, J=5.0 \mathrm{~Hz}, 1 \mathrm{H}, 5$-thienyl $\mathrm{H}), 7.19(\mathrm{t}, J=4.4 \mathrm{~Hz}, 1 \mathrm{H}, 4$-thienyl H), $7.00(\mathrm{~d}, J=3.2 \mathrm{~Hz}, 1 \mathrm{H}, 3$-thienyl H), $2.65(\mathrm{~s}, 6 \mathrm{H}$, 3,5- $\left.\mathrm{CH}_{3}\right), 1.59\left(\mathrm{~s}, 6 \mathrm{H}, 1,7-\mathrm{CH}_{3}\right) ;{ }^{13} \mathrm{C} \mathrm{NMR}\left(100 \mathrm{MHz}, \mathrm{CDCl}_{3}\right) \delta 157.35,145.65,134.34$, 133.75, 132.25, 128.20, 128.14, 127.97, 86.17, 16.27, 16.11; HRMS (ESI-TOF) $\mathrm{m} / \mathrm{z}$ $582.9173[\mathrm{M}+\mathrm{H}]^{+}$, calculated for $\mathrm{C}_{17} \mathrm{H}_{16} \mathrm{BF}_{2} \mathrm{I}_{2} \mathrm{~N}_{2} \mathrm{~S}: 582.9185$.

\subsubsection{BODIPY 8a (4,4-difluoro-2,6-di-iodo-8-([2,2'-bithiophen]-2-yl)-1,3,5,7-} tetramethyl-4-bora-3a, 4a-diaza-s-indacene): Obtained as a red solid $(0.5073 \mathrm{~g})$ in $84 \%$ yield from $8(0.250 \mathrm{~g}, 0.6063 \mathrm{mmol})$, iodic acid $(0.2133 \mathrm{~g}, 1.2126 \mathrm{mmol})$, iodine $(0.1924 \mathrm{~g}$, $1.5158 \mathrm{mmol}), \mathrm{mp}=195-196{ }^{\circ} \mathrm{C} ;{ }^{1} \mathrm{H} \mathrm{NMR}\left(400 \mathrm{MHz}, \mathrm{CDCl}_{3}\right) \delta 7.25\left(\mathrm{t}, J=3.3 \mathrm{~Hz}, 1 \mathrm{H}, 5^{\prime}-\right.$ bithienyl H), $7.21(\mathrm{~d}, J=3.9 \mathrm{~Hz}, 1 \mathrm{H}, 4$-bithienyl H), $7.18(\mathrm{~d}, J=3.4 \mathrm{~Hz}, 1 \mathrm{H}, 3$-bithienyl H), $6.91\left(\mathrm{dd}, J=5.1\right.$ and $4.2 \mathrm{~Hz}, 2 \mathrm{H}, 3^{\prime}, 4^{\prime}$-bithienyl), $2.66\left(\mathrm{~s}, 6 \mathrm{H}, 3,5-\mathrm{CH}_{3}\right), 1.74(\mathrm{~s}, 6 \mathrm{H}, 1,7-$ $\left.\mathrm{CH}_{3}\right) ;{ }^{13} \mathrm{C} \mathrm{NMR}\left(100 \mathrm{MHz}, \mathrm{CDCl}_{3}\right) \delta 157.67,145.50,141.83,139.22,137.88,133.23$, 129.13, 129.04, 128.09, 125.95, 125.41, 124.67, 86.28, 16.69, 16.14; HRMS (ESI-TOF) $\mathrm{m} / \mathrm{z}$ $663.8988[\mathrm{M}+\mathrm{H}]^{+}$, calculated for $\mathrm{C}_{21} \mathrm{H}_{17} \mathrm{BF}_{2} \mathrm{I}_{1} \mathrm{~N}_{2} \mathrm{~S}_{2}: 663.8984$.

\subsubsection{BODIPY 9a (4,4-Difluoro-8-(5-bromothiophen-2-yl)-2,6-di-iodo-1,3,5,7-} tetramethyl-4-bora-3a,4a-diaza-s-indacene): Obtained as a red solid $(0.0738 \mathrm{~g})$ in $91 \%$ yield from $9(0.050 \mathrm{~g}, 0.1222 \mathrm{mmol})$, iodic acid $(0.0430 \mathrm{~g}, 0.2444 \mathrm{mmol})$, iodine $(0.0388 \mathrm{~g}$, $0.3055 \mathrm{mmol}), \mathrm{mp}=212{ }^{\circ} \mathrm{C}$ (decomposes); ${ }^{1} \mathrm{H} \mathrm{NMR}\left(400 \mathrm{MHz}, \mathrm{CDCl}_{3}\right) \delta 7.15$ (d, $J=3.4$ $\mathrm{Hz}, 1 \mathrm{H}, 4$-thienyl-H), 6.79 (d, $J=3.9 \mathrm{~Hz}, 1 \mathrm{H}, 3$-thienyl-H), 2.65 (s, 6H, 3,5- $\mathrm{CH}_{3}$ ), 1.71 (s, $\left.6 \mathrm{H}, 1,7-\mathrm{CH}_{3}\right) ;{ }^{13} \mathrm{C}$ NMR $\left(100 \mathrm{MHz}, \mathrm{CDCl}_{3}\right) \delta 158.18,145.48,136.18,132.63,130.65$, $128.79,114.84,86.05,16.63,16.15$; HRMS (ESI-TOF) $\mathrm{m} / \mathrm{z} 660.8246[\mathrm{M}+\mathrm{H}]^{+}$, calculated for $\mathrm{C}_{17} \mathrm{H}_{15} \mathrm{BBrF}_{2} \mathrm{I}_{2} \mathrm{~N}_{2} \mathrm{~S}: 660.8290$.

\subsubsection{BODIPY 10a (4,4-Difluoro-8-(5'-bromo-[2,2'-bithiophen]-2-yl)-2,6-} diiodo-1,3,5,7-tetramethyl-4-bora-3a,4a-diaza-s-indacene): Obtained as a red solid 
$(0.2457 \mathrm{~g})$ in $65 \%$ yield from $10(0.250 \mathrm{~g}, 0.5089 \mathrm{mmol})$, iodic acid $(0.1791 \mathrm{~g}, 1.0179$ $\mathrm{mmol}$ ), iodine (0.1615 g, $1.2723 \mathrm{mmol}), \mathrm{mp}=198{ }^{\circ} \mathrm{C}$ (decomposes); ${ }^{1} \mathrm{H}$ NMR $(400 \mathrm{MHz}$, $\left.\mathrm{CDCl}_{3}\right) \delta 7.17\left(\mathrm{~d}, J=3.5 \mathrm{~Hz}, 1 \mathrm{H}, 4,4^{\prime}\right.$-bithienyl-H), 7.03 (d, $J=3.9 \mathrm{~Hz}, 1 \mathrm{H}, 4,4^{\prime}$-bithienyl H), 6.98 (d, $J=3.9 \mathrm{~Hz}, 1 \mathrm{H}, 3,3^{\prime}$-bithienyl-H), 6.90 (d, $J=3.5 \mathrm{~Hz}, 1 \mathrm{H}, 3,3^{\prime}$-bithienyl-H),

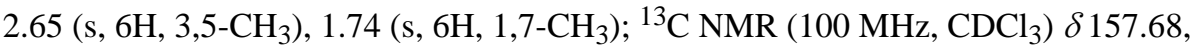
$145.50,139.38,137.40,133.20,132.42,132.16,130.85,129.13,124.75,124.14,112.25$, $86.32,16.68,16.15$; MS (MALDI-TOF) $m / z 741.8490[\mathrm{M}+\mathrm{H}]^{+}$, calculated for $\mathrm{C}_{21} \mathrm{H}_{16} \mathrm{BBrF}_{2} \mathrm{I}_{2} \mathrm{~N}_{2} \mathrm{~S}_{2}: 741.8089$.

2.1.2.11. BODIPY 11a (4,4-Difluoro-8-pentafluorophenyl-2,6-di-iodo-1,3,5,7tetramethyl-4-bora-3a,4a-diaza-s-indacene): Obtained as a red solid $(0.0726 \mathrm{~g})$ in $90 \%$ yield from $11(0.050 \mathrm{~g}, 0.1207 \mathrm{mmol})$, iodic acid $(0.0425 \mathrm{~g}, 0.2415 \mathrm{mmol})$, iodine $(0.0383 \mathrm{~g}$, $0.3018 \mathrm{mmol}), \mathrm{mp}=198{ }^{\circ} \mathrm{C}$ (decomposes); ${ }^{1} \mathrm{H}$ NMR $\left(400 \mathrm{MHz}, \mathrm{CDCl}_{3}\right) \delta 2.68(\mathrm{~s}, 6 \mathrm{H}, 3,5-$ $\left.\mathrm{CH}_{3}\right), 1.65\left(\mathrm{~s}, 6 \mathrm{H}, 1,7-\mathrm{CH}_{3}\right) ;{ }^{13} \mathrm{C} \mathrm{NMR}\left(100 \mathrm{MHz}, \mathrm{CDCl}_{3}\right) \delta 159.13,145.11,143.74$, 142.70, 139.65, 137.07, 130.89, 122.46, 87.05, 16.31, 16.20; HRMS (ESI-TOF) $\mathrm{m} / \mathrm{z}$ $666.9130[\mathrm{M}+\mathrm{H}]^{+}$, calculated for $\mathrm{C}_{19} \mathrm{H}_{13} \mathrm{BF}_{7} \mathrm{I}_{2} \mathrm{~N}_{2}: 666.9150$.

\subsection{Molecular structures}

The crystal structures of BODIPYs 3, 7, 1a and 9a were determined at low temperature using MoKa radiation on a Nonius KappaCCD (1a) diffractometer, CuKa radiation on a Bruker Kappa Apex-II (3), or CuKa (7) or MoKa (9a) radiation on a Bruker Kappa Apex-II DUO diffractometer. For $\mathbf{7}$, the thiophene substituent was disordered into two orientations with 0.824(2)/0.176(2) occupancies, and for 9a, there were two independent molecules in the asymmetric unit. Crystal data: $1 \mathrm{a}, \mathrm{C}_{19} \mathrm{H}_{17} \mathrm{BF}_{2} \mathrm{I}_{2} \mathrm{~N}_{2}, M=575.96$, monoclinic, $a=$ $11.4673(15), b=12.9564(15), c=17.3920(15) \AA, \beta=130.446(4)^{\circ}, U=1966.5(4) \AA^{3}, T=$ $95 \mathrm{~K}$, space group $\mathrm{P} 2_{1} / c, Z=4,16,872$ reflections measured, 8604 unique $\left(R_{\text {int }}=0.032\right)$ which were used in all calculations. The final $R=0.031$ (6345I>2o(I) data), $w R\left(F_{2}\right)=$ 0.060 (all data), CCDC 929873; 3, $\mathrm{C}_{27} \mathrm{H}_{35} \mathrm{BF}_{2} \mathrm{~N}_{2}, M=436.38$, monoclinic, $a=6.6395(8)$, $b=12.1074(14), c=15.139(2) \AA, \beta=101.284(10)^{\circ}, U=1193.5(3) \AA^{3}, T=90 \mathrm{~K}$, space group $P 2 / c, Z=2,9239$ reflections measured, 2097 unique $\left(R_{\mathrm{int}}=0.042\right)$, final $R=0.036$ $\left(1887 I>2 \sigma(I)\right.$ data), $w R\left(F_{2}\right)=0.094$ (all data), CCDC 929871; 7, $\mathrm{C}_{17} \mathrm{H}_{17} \mathrm{BF}_{2} \mathrm{~N}_{2} \mathrm{~S}, M=$ 330.20, monoclinic, $a=6.6059(3), b=18.5456(9), c=12.7397(6) \AA, \beta=92.791(2)^{\circ}, U=$ 1558.89(13) $\AA^{3}, T=90 \mathrm{~K}$, space group $\mathrm{P} 2{ }_{1} / c, Z=4,14,155$ reflections measured, 2797 unique $\left(R_{\text {int }}=0.035\right)$, final $R=0.034\left(2762 I>2 \sigma(I)\right.$ data), $w R\left(F_{2}\right)=0.080$ (all data), CCDC 929872; 9a, $\mathrm{C}_{17} \mathrm{H}_{14}-\mathrm{BBrF}_{2} \mathrm{I}_{2} \mathrm{~N}_{2} \mathrm{~S}, M=660.88$, monoclinic, $a=21.871(2), b=9.8112(10), c$ $=19.478(2) \AA, \beta=107.585(5)^{\circ}, U=3984.3(7) \AA^{3}, T=100 \mathrm{~K}$, space group $\mathrm{P} 2_{1} / c, Z=8$, 162,698 reflections measured, 26,346 unique $\left(R_{\text {int }}=0.052\right)$, final $R=0.029(19,684 I>2 \sigma(I)$ data), $w R\left(F_{2}\right)=0.060$ (all data), CCDC 934829 .

\subsection{Spectroscopic studies}

The photophysical properties of all compounds were determined on solutions prepared by dissolving an adequate amount of crystalline compound in either dichloromethane or tetrahydrofuran. Stock solutions of concentrations between $1.5 \times 10^{-5}$ and $5 \times 10^{-5} \mathrm{M}$ were prepared and diluted to appropriate concentrations for collection of absorbance and emission spectra. 
Absorption spectra were acquired using a PerkinElmer Lambda 35 UV/vis spectrometer. Measurements obtained for determining optical density, $\varepsilon$, were taken from prepared solutions with concentrations between $7.5 \times 10^{-6}$ and $2.5 \times 10^{-5}$ Min order to obtain $\lambda_{\max }$ between 0.5 and1.0. Fluorescence spectra were recorded by further dilution of stock solutions to between $1.5 \times 10^{-6}$ and $8 \times 10^{-6}$ Mto achieve an optical density at the excitation wavelength between 0.04 and 0.06 to minimize intermolecular reabsorption and inner-filter effects. ${ }^{38}$ Emission measurements were chronicled on a PTI QuantaMaster4/2006SE spectrofluorometer with the slit width set at $2 \mathrm{~nm}$. Rhodamine 6G was used as a standard in calculating the fluorescence quantum yields ( $\phi_{\mathrm{F}}=0.95$ in ethanol). Fluorescence emissions were recorded for all samples, including the standard, after excitation at $480 \mathrm{~nm}$. All measurements, both absorbance and emission, were acquired within $4 \mathrm{~h}$ of solution preparation at room temperature $\left(23-25^{\circ} \mathrm{C}\right)$, using a $10 \mathrm{~mm}$ path length spectrophotometric cell.

The fluorescent quantum yields $\left(\Phi_{\mathrm{f}}\right)$ were calculated using the following equation: ${ }^{39}$

$$
\Phi_{\text {exp }}=\Phi_{\text {ref }} \times \frac{F_{x}\left[A_{\text {std }}\right] n_{x}^{2}}{F_{\text {std }}\left[A_{x}\right] n_{\text {std }}^{2}}
$$

$\Phi_{\text {ref }}$ is the fluorescent quantum yield of the standard, $F_{x}$ is the area under the sample's emission peak, $F_{\text {std }}$ is the area under the standard's emission peak, $A_{\text {std }}$ is the optical density at which the standard was excited, $A_{x}$ is the optical density at which the sample was excited, $\mathrm{n}$ is the refractive index of the sample's solvent, and $n_{\text {std }}$ is the refractive index of the standard's solvent.

\subsection{Computational modeling}

Electronic structure calculations of BODIPYs 1-11 and 1a-11a were carried out using the hybrid Becke's Three Parameter DFT Functional. ${ }^{40,41}$ All atoms except iodine were modeled using the $6-31+\mathrm{G}(\mathrm{d}, \mathrm{p})$ basis sets. For BODIPY's 1a-11a the iodine atoms were treated using the Stevens-Basch-Krauss (SBK) ${ }^{42-44}$ relativistic effective core potentials and the standard CEP-31G basis set. All structures were optimized without symmetry constraints. The Highest Occupied Molecular Orbital (HOMO) and the Lowest Unoccupied Molecular Orbital (LUMO) were rigorously determined without any further approximations. Potential energy surface minima were confirmed with frequency calculations. Rotational energy barriers were determined by performing relaxed scans of the potential energy surface. All calculations were performed using the GAUSSIAN 09 program package. ${ }^{45}$

\subsection{Cell studies}

The human HEp2 cells used in this study were purchased from ATCC (derived from HeLa, cervical cancer, contamination). The HEp2 cells were maintained in a 50:50 mixture of DMEM:AMEM (Invitrogen) supplemented with 10\% FBS (Invitrogen) and 1\% antibiotic (penicillin-streptomycin) and $5 \% \mathrm{CO}_{2}$ at $37^{\circ} \mathrm{C}$. A $32 \mathrm{mM} \mathrm{BODIPY}$ stock solution was prepared by dissolving the compound in $96 \%$ DMSO and $4 \%$ Cremophor EL (a nonionic emulsifier). A $2 \mathrm{~mL}$ of a $400 \mu \mathrm{M}$ BODIPY solution containing 1.95\% DMSO and $0.05 \%$ 
Cremophor EL was prepared by adding $15 \mu \mathrm{L}$ DMSO and $25 \mu \mathrm{L}$ of the $32 \mathrm{mM}$ stock solution into $1960 \mu \mathrm{L}$ medium. The final solution was sonicated to aid in BODIPY solubilization.

2.5.1. Time-dependent cellular uptake-The HEP 2 cells were plated at 15,000 cells per well in a Costar 96-well plate (BD biosciences) and grown overnight. The $10 \mu \mathrm{M}$ BODIPY solution was prepared by diluting $400 \mu \mathrm{M}$ stock solution with medium containing $5 \%$ FBS and $1 \%$ antibiotic. The cells were treated by adding $100 \mu \mathrm{L} / \mathrm{well}$ of the $10 \mu \mathrm{M}$ BODIPY solution at time periods of $0,1,2,4,8$, and $24 \mathrm{~h}$. The loading medium was removed at the end of the treatments. The cells were washed with 1X PBS, and solubilized by adding $0.25 \%$ Triton X-100 in $1 \mathrm{X}$ PBS. BODIPY standard curves at 10, 5, 2.5, 1.25, 0.625 and $0.3125 \mu \mathrm{M}$ concentrations were obtained by diluting $20 \mathrm{mM}$ BODIPY solution with $0.25 \%$ Triton X-100 in $1 \times$ PBS. A cell standard curve was prepared using 10,000, $20,000,40,000,60,000,80,000$, and 100,000 cells per well. The cells were quantified using theCyQuant Cell Proliferation Assay (Life Technologies). The compound and cell number were determined using a FluoStar Optima micro-plate reader (BMG LRBTEH), with wavelengths $355 / 520$ and $485 / 520 \mathrm{~nm}$. Cellular uptake was expressed in terms of $\mathrm{nM}$ compound per cell.

2.5.2. Dark cytotoxicity-The HEp2 cells were placed in a 96-well plate as above, with BODIPY concentrations of 400, 200,100, 50, 25, 12.5, and $0 \mu \mathrm{M}$, five repetitions for each concentration, and then incubated at $37^{\circ} \mathrm{C}$. After $24 \mathrm{~h}$ incubation, the compound was removed by washing the cells with $1 \mathrm{X}$ PBS and replaced with media containing $20 \%$ Cell Titer Blue. The cells were incubated for an additional $4 \mathrm{~h}$ at $37^{\circ} \mathrm{C}$. The viable cells were measured using fluorescence at 570/615 nm using a FluoStar Optima micro-plate reader. The dark toxicity was expressed in terms of the percentage of viable cells.

2.5.3. Phototoxicity-The concentration range of $200,100,50,25,12.5,6.25,3.125$ and $0 \mu \mathrm{M}$ was used for the phototoxicity experiments. The HEp2 cells were placed in 96-well plates as described above, and treated with compound for $24 \mathrm{~h}$ at $37^{\circ} \mathrm{C}$. After the $24 \mathrm{~h}$ treatment, the loading media was removed. The cells were washed with media, and then refilled with fresh media. The cells were placed on ice and exposed to $610 \mathrm{~nm}$ LP filter light from a 100W halogen lamp filtered through a $610 \mathrm{~nm}$ long pass filter (Chroma) for $20 \mathrm{~min}$. An inverted plate lid filled with cold water to a depth of $5 \mathrm{~mm}$ acted as an IR filter. The total light dose was approximately $1.5 \mathrm{~J} / \mathrm{cm}^{2}$. After exposure to light, the cells were returned back to the incubator for $24 \mathrm{~h}$. After $24 \mathrm{~h}$ incubation, the medium was removed and replaced with medium containing $20 \%$ of Cell Titer Blue. The cells were incubated for an additional $4 \mathrm{~h}$. The viable cells were measured by fluorescence at 570/615 nm using a FluoStar Optima micro-plate reader. The phototoxicity was expressed in terms of the percentage of viable cells.

2.5.4. Comparative singlet oxygen quantum yields-To each well of a 6-well plate was added $2 \mathrm{~mL}$ containing $50 \mu \mathrm{M}$ of 1,3-diphenylisobenzofuran (DPBF) and $5 \mu \mathrm{M}$ of each photosensitizer in DMSO. The plate was irradiated using a $71 \mathrm{~W}$ filtered light source of $>500$ $\mathrm{nm}$ with a Schott glass $500 \mathrm{~nm}$ long-pass yellow filter for $1 \mathrm{~h}$. At 15 min increments, $200 \mu \mathrm{L}$ 
aliquots were removed from each of the six wells and the absorbance was measured at 410 $\mathrm{nm}$. The rate of singlet oxygen generation was determined by the decrease in absorbance of DPBF over time. Control solutions of DPBF-DMSO (negative control) and DPBFmethylene blue-DMSO (reference standard) were irradiated under the previous mentioned conditions. Singlet oxygen quantum yields were determined using the following equation:

$$
\Phi_{\Delta(x)}=\Phi_{\Delta(\mathrm{std})} \times \frac{S_{x}}{S_{\text {std }}}
$$

$\Phi_{\Delta(U)}$ is the singlet oxygen quantum yield of the sample, $\Phi_{\Delta(\mathrm{std})}$ is the singlet oxygen quantum yield of the standard (methylene blue, 0.52 ), $S_{U}$ is the slope of the plot of absorbance versus time of the sample, and $s_{\text {std }}$ is the slope of the plot of absorbance versus time of the standard. ${ }^{15}$

\section{Results and discussion}

\subsection{Synthesis and structural characterization}

Eleven meso-aryl BODIPYs (1-11) were synthesized from commercially available 2,4dimethylpyrrole and the corresponding aryl aldehyde, following a three-step one-pot procedure often used for BODIPY synthesis ${ }^{46}$ (Scheme 1). First, two pyrrole units were condensed with the aryl aldehyde in dichloromethane in the presence of $\mathrm{BF}_{3} \cdot \mathrm{OEt}_{2}$, then the resulting dipyrromethanes were oxidized using DDQ and finally the dipyrromethenes were complexed with excess $\mathrm{BF}_{3} \cdot \mathrm{OEt}_{2}$ under basic conditions. Following work-up, the BODIPYs were purified by silica gel column chromatography and re-crystallized, to give the target compounds in 18 to $58 \%$ yields. All starting aldehydes are commercially available except $5^{\prime}$ bromo-[2,2'-bithiophen]-5-carbaldehyde that was used in the synthesis of BODIPY $\mathbf{1 0}$, which was prepared from [2,2'-bithiophen]-5-carbaldehyde in $64 \%$ yield, using a published procedure. ${ }^{25}$ Subsequent iodination of BODIPYs 1-11 to produce 1a-11a was accomplished through electrophilic substitution at the 2,6-positions using iodic acid in ethanol and dichloromethane at $60{ }^{\circ} \mathrm{C}$ for $2 \mathrm{~h}$, in $57-94 \%$ yield. ${ }^{13,15}$ These meso-aryl BODIPYs were synthesized to investigate the effects of halogenation and the nature of the meso-aryl groups on the photophysical and cytotoxic properties of the BODIPYs. MesoSubstitution has been observed to increase the photostability of the BODIPY ${ }^{47,48}$ and iodination at the 2,6-positions is reported to enhance intersystem crossing by the "heavy atom effect,' and consequently the phototoxicity of the BODIPYs. ${ }^{13-15}$ On the other hand, halogenation at the 3,5-positions or at the BODIPY meso-phenyl or meso-thienyl substituents is not expected to significantly affect the cytotoxicity of the compounds.

All BODIPYs were characterized by ${ }^{1} \mathrm{H}$ and ${ }^{13} \mathrm{C}$ NMR, HRMS and, in the case of $\mathbf{3}, \mathbf{7}, \mathbf{1 a}$, and 9a, by X-ray crystallography (Fig. 1). Analysis of the NMR spectra (see Supplementary data, Figs. S11-S31) shows the twofold symmetry of the BODIPY core. Single peaks for the 2,6-hydrogens, the 1,7- and 3,5-dimethyl groups on the ${ }^{1} \mathrm{H}$ NMR spectra and the appearance of signals for only half of the BODIPY core's carbons in ${ }^{13} \mathrm{C}$ NMR, indicate a plane of symmetry extending through the boron and carbon-8 (meso-position). The upfield shift of the 1,7-dimethyl groups (at approximately $1.6 \mathrm{ppm}$ ) compared with the 3,5-dimethyls (at 
approximately $2.5 \mathrm{ppm}$ ) is in part due to the shielding by the meso-aryl groups. The disappearance of the 2,6-hydrogens (at $\sim 6.0 \mathrm{ppm}$ ) in the ${ }^{1} \mathrm{H}$ NMR spectra indicated complete iodination; additional evidence was provided by the shift of the carbon atoms bearing these hydrogens in ${ }^{13} \mathrm{C}$ NMR, from 120 to $85 \mathrm{ppm}$.

Single crystals of two $\beta$-free and two di-iodo BODIPYs suitable for X-ray analyses were grown from dichloromethane, acetone, or chloroform- $d$ and their molecular structures are shown in Figure 1. X-ray analyses reveal the expected approximate twofold symmetry of all four compounds, with two conformers of BODIPYs $\mathbf{7}$ and $\mathbf{9 a}$ being elucidated (only one of each is shown in Fig. 1). This suggests that the thienyl group located at the meso-position has greater rotational freedom compared with the meso-phenyl substituent. The BODIPY core and meso-substituent lie nearly perpendicular to one another in the molecule's most relaxed form, which reduces the steric strain caused by the 1,7-dimethyl groups. ${ }^{49}$ The boron possess nearly tetrahedral geometry with the two fluorines lying perpendicular to the BODIPY core.

In comparison to previously reported boron-dipyrromethene compounds, the BODIPY cores of 1a, 3, 7 and 9a adopt expected bond lengths, planarity, and orthogonal dihedral angles of the $\mathrm{F}$ atoms relative to the $\mathrm{C}_{9} \mathrm{~N}_{2} \mathrm{~B}$ (excluding peripheral $\mathrm{H}$ atoms and substituents) aromatic framework. The dihedral angles of the meso-substituents are also nearly $90^{\circ}$ out-of-plane of the BODIPY core in the four crystal structures $\left(\mathbf{1 a} 88.01(5)^{\circ} ; \mathbf{3} 78.60(4)^{\circ} ; \mathbf{7}\right.$ two partially occupied orientations: $82.8(2)^{\circ}$ and $84.19(11)^{\circ}$; 9a two independent molecules $82.83(3)^{\circ}$ and 89.54 $\left.(2)^{\circ}\right)$. Intermolecular halogen-halogen bonding exist in the iodinated BODIPYs 1a

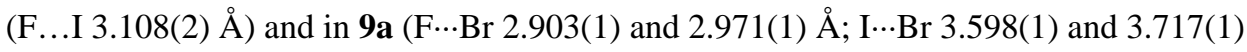

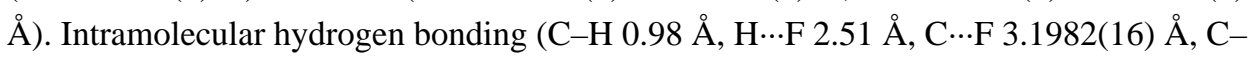
$\mathrm{H} \cdots \mathrm{F} 127^{\circ}$ ) between the $\mathrm{F}$ atoms with a $\mathrm{H}$ atom of a methyl group (on the BODIPY core's a-carbon site) is present in BODIPY 3 .

For BODIPY 7, the average structure contains two partially occupied orientations. The orientations can be modeled using static (positional) disorder with a refined occupancy ratio of 0.824(2):0.176(2) for two of the thiophene atoms ( $\mathrm{S}$ and one of the $\mathrm{C}$ atoms). In Figure 1, only one of the two orientations is shown. The crystal structure of BODIPY 3 has been previously reported in literature and Cambridge Structural Database (CCDC 712038) based on room temperature measurements. ${ }^{29}$ Other close matches can be found for two other crystal structures (1a and 7) determined in this study. These close matches (CCDC 812643 and 856179) have been characterized by others and are extensions of $1 \mathbf{a}$ (with a mesomesityl group, instead of a phenyl) and $\mathbf{7}$ (with additional quinolin-2-yl on the thiophene's other a-carbon atom). ${ }^{50,51}$

The symmetric nature of the BODIPYs shown by NMR and X-ray crystallography were confirmed by computational modeling. Although no symmetry constraints were used in the calculations and the starting geometries were not symmetric, the optimized geometries of BODIPYs are nearly symmetric (e.g., $\mathrm{C}_{2}$ for BODIPY $\mathbf{3}$ and $\mathrm{C}_{\mathrm{s}}$ for BODIPY 7). The BODIPY core and meso-substituent form angles between $89.9^{\circ}$ and $90.1^{\circ}$. 


\subsection{Spectroscopic properties and computational studies}

The spectroscopic properties of BODIPYs 1-11 and 1a-11a were evaluated in dichloromethane and THF, and the results obtained are summarized in Table 1 and also shown in Supplementary data, Figures S1-S6. Little to no solvent effect was observed for these two solvents in the absorption and emission maxima wavelengths. Such lack of influence by the polarity of the solvent indicates that the permanent dipole moments of the BODIPYs do not change between the ground state and the excited state ${ }^{52}$ The synthesized $\beta$-free, meso-aryl BODIPYs display absorptions between $499<\lambda_{\text {abs }}<517 \mathrm{~nm}$ and emission bands between $507<\lambda_{\mathrm{em}}<530 \mathrm{~nm}$. BODIPYs 1-11 show typical fluorescence in the green/ yellow spectral region with fluorescence quantum yields in the range 0.03-1.0. The mesothienyl- and pentafluorophenyl BODIPYs exhibited the largest red-shifted profiles and the lowest quantum yields of this series of compounds due to the presence of the sulfur and five fluorines, respectively, which impact the conjugation of the BODIPY $\pi$-system (see below).

Incorporation of iodines onto the BODIPY core, as in compounds 1a-11a, causes a shift in the absorption and emission profiles to longer wavelengths, into the orange/red spectral region, and decrease the fluorescence quantum yields, as previously observed. ${ }^{13-15}$ The trend observed in the $\beta$-free BODIPYs is also seen in their di-iodo analogs with absorption and emisson profiles becoming more red-shifted with the incorporation of thienyl- and pentafluorophenyl-groups. All compounds displayed high extinction coefficients, in the order of 23,000-118,000 $\mathrm{M}^{-1} \mathrm{~cm}^{-1}$ ( $\log \varepsilon$ values between 4.34 and 5.07), and Stokes' shifts in the range 5-20 nm. BODIPYs 7-11 and 7a-11a, bearing meso-thienyl and pentafluorophenyl substituents, displayed greater red-shifted absorptions and emissions (by 13-41 nm) compared with the other meso-phenyl BODIPYs 1-6 and 1a-6a. The electronwithdrawing effects of the $\mathrm{S}$ and the five $\mathrm{F}$ atoms on the meso-substituent of BODIPYs 7-11 and 7a-11a tend to stabilize the LUMO via delocalization of the electron density (see below). ${ }^{53}$ This causes a decrease in the energy of the LUMO, decreasing the HOMOLUMO gap, and therefore increasing the absorbance and emission maxima wavelengths.

This effect was examined computationally (details of the theoretical level are given the Section 2). The calculated HOMO-LUMO gaps are listed in Table 2, along with the HOMO and LUMO energies. Indeed, for BODIPYs 5-11 the LUMO decreases gradually. However, this is not the sole effect determining the gap. In addition to LUMO lowering, the HOMO varies substantially. Therefore, the combination of the HOMO and LUMO effects determine the observed trend. In general, the computational models predict that the eleven BODIPYs will be grouped into three groups: BODIPYs 1-6 with gaps around $3.0 \mathrm{eV}$, BODIPYs 7-11 with gaps around $2.9 \mathrm{eV}$, and BODIPY 10a with significantly lower gap of $2.7 \mathrm{eV}$ (see Fig. 2). Similar tendencies are seen for the di-iodo BODIPYs; BODIPYs 1a-6a with gaps around $2.9 \mathrm{eV}$, BODIPYs 7a-11a with gaps around $2.8 \mathrm{eV}$, and BODIPY 10a having again significantly lower gap of $2.7 \mathrm{eV}$. This theoretical prediction is in excellent agreement with the experimentally measured red-shifts of the meso-thienyl-BODIPYs 7-10 and the mesopentafluorophenyl-BODIPY 11, and the most pronounced red-shift of the di-iodo-BODIPY 10a. In general, the di-iodo substitution lowers both the HOMO and LUMO, with the effect on LUMO being slightly more emphasized. The overall effect is to lower slightly the 
HOMO-LUMO gap, which is in excellent agreement with the experimentally observed redshifts for all di-iodo BODIPYs when compared with their $\beta$-free analogs.

It was also observed that the fluorescence quantum yields for the meso-phenyl BODIPYs 1$\mathbf{6}$ and $\mathbf{1 1}\left(0.4<\phi_{\mathrm{F}}<1.0\right)$ were greater than for the meso-thienyl BODIPYs 7-10 $\left(0.04<\phi_{\mathrm{F}}\right.$ $<0.12$ ). Due to the smaller size of the meso-group, the thienyl substituent has greater freedom of rotation, which increases the amount of energy lost to non-radiative decay. This spinning motion increases the energy of the system which, in turn, decreases the number of photons that become excited and relax via fluorescence. Negligible effects were caused by the change of solvent. Nevertheless, these results suggest that the meso-phenyl BODIPYs with appropriate functionalization may serve as significantly brighter fluorophores in aqueous media than the meso-thienyl derivatives. Furthermore, incorporating a longer chain group in the meso-position increases the degree of rotational freedom, which also decreases the fluorescence quantum yield as indicated by the results obtained for BODIPYs 7 ( $\phi_{\mathrm{F}}=$ $0.09-0.11)$ and $\mathbf{8}\left(\phi_{\mathrm{F}}=0.03-0.04\right)$.

Addition of bromine onto the meso-thienyl group has only a slight effect on the fluorescence quantum yield, as observed for BODIPYs $7\left(\phi_{\mathrm{F}}=0.11\right)$ and $\mathbf{9}\left(\phi_{\mathrm{F}}=0.12\right)$ in dichloromethane. This result suggests that addition of heavy atoms, such as bromine, onto the meso-substituent has little effect on the fluorescence quantum yield, while incorporation of iodines at the 2,6-positions of the BODIPY core, and of meso-thienyl groups, significantly decrease the fluorescence quantum yields. With the exception of the fluorines on the meso-pentafluorophenyl-BODIPY 11, the quantum yields significantly decline (1a11a, $\left.0.01<\phi_{\mathrm{F}}<0.07\right)$.

The rotational freedom of different meso-substituents in vacuum and in dichloromethane was studied computationally at the B3LYP/6-31+G (d,p) level for BODIPYs 1-11. The energy barriers for the rotation of the meso-aryl group are given in the last column of Table 2. For most compounds, there is a clear correlation between the rotational barrier and the fluorescence quantum yield (Fig. 3). The meso-thienyl group in BODIPYs 7-10 can easily rotate with a barrier of only $14-16 \mathrm{kcal} / \mathrm{mol}$, which explains the low quantum yield observed for this series of compounds. Heavy atom substitution does not have significant effect on the rotational barrier, which is in agreement with the negligible changes observed in the quantum yield. Taking the solvent into account slightly lowers the rotational barriers but the effect is small and the tendency remains very similar.

BODIPYs $\mathbf{1}$ and $\mathbf{5}$ exhibit intermediate rotational barriers and intermediate fluorescence quantum yields. Having a meso-phenyl group with substituents at the meta-position $\left(\mathrm{OCH}_{3}\right.$ or $t \mathrm{Bu}$ ) hinders the rotation and results in significantly higher quantum yields, compared with substituents at the para-position. BODIPYs $2,3,4$, and $\mathbf{6}$ experience rotational barriers of 20-24 kcal/mol, which is comparable to the kinetic energy at room temperature. The $\mathrm{F}_{5}-$ substituted BODIPY 11 deviates from the relationship to the highest extent probably due to the strong electron-withdrawing character of the meso-pentafluorophenyl group. Still, it shows the highest rotational barrier and a large fluorescence quantum yield. 


\subsection{Cellular properties}

The cytotoxicity of a select group of $\beta$-free BODIPYs $(\mathbf{5}, \mathbf{7}, \mathbf{8}$ and $\mathbf{1 0})$ and of all 2,6-di-iodoBODIPYs were investigated in human HEp2 cells using the Cell Titer Blue assay, and the results obtained are summarized in Table 3 (see also Figs. S7-S10 of the Supplementary data). All but one BODIPY (10a, $\left.\mathrm{IC}_{50}=8 \mu \mathrm{M}\right)$ were found to be non-toxic in the dark, with determined $\mathrm{IC}_{50}$ values, from dose-response curves, above $400 \mu \mathrm{M}$. Upon exposure to a low light dose $\left(1.5 \mathrm{~J} / \mathrm{cm}^{2}\right)$ all the $\beta$-free BODIPYs investigated showed low cytotoxicity $\left(\mathrm{IC}_{50}\right.$ $>80 \mu \mathrm{M}$ ) in agreement with previous investigations. ${ }^{15,48}$ Among the 2,6-diiodo-BODIPYs, 1a, 2a, 4a, 6a, 7a and 10a showed $\mathrm{IC}_{50}$ values between 3.5 and $28 \mu \mathrm{M}$, while all others (3a, 5a, 8a, 9a and 11a) showed $\mathrm{IC}_{50}>200 \mu \mathrm{M}$. This is a surprising result, since the 2,6-diiodoBODIPYs previously investigated are reported to have high phototoxicity, ${ }^{14,15}$ attributed to the 'heavy atom effect,' and cleary shows the effect of the meso-aryl groups. In particular, BODIPYs 2a, 4a and 10a bearing meso-dimethoxyphenyl or bromo-bithienyl substituents show the highest phototoxicity $\left(\mathrm{IC}_{50}=3.5-7.5 \mu \mathrm{M}\right.$ at $1.5 \mathrm{~J} / \mathrm{cm}^{2}$ ) and moderate relative rates of singlet oxygen generation (see below); among these, BODIPYs $\mathbf{2 a}$ and $\mathbf{4 a}$ are the most promising for PDT applications due to their high dark/phototoxicity ratio (>50). The observed high phototoxicity of BODIPY 10a is likely attributed to its substantial dark toxicity. On the other hand, BODIPYs 3a, 5a, 8a, 9a and 11a showed no dark/photo cytotoxicities and could therefore find application as radioiodine-labeled imaging agents for SPECT and PET, provided they are deemed to possess specific cellular targeting attributes not yet investigated.

The high dark and phototoxicity observed for BODIPY 10a might be in part due to its remarkably higher cellular uptake, as shown in Figure 4. On the other hand, BODIPYs 2a and $4 \mathbf{a}$ were the least accumulated within HEp2 cells, although they were also highly phototoxic, which might be due to their binding to certain protein lipophilic sites. ${ }^{54}$ Preliminary results (not shown) reveal that all the phototoxic BODIPYs (1a, 2a, 4a, 6a, 7a and 10a) localized subcellularly partly within mitochondria, the cell 'power house'. ${ }^{55} \mathrm{In}$ particular, the role played by mitochondria in apoptosis, the process of programmed cell death, makes these cellular organelles highly desirable targets for PDT. ${ }^{56}$ These results are in agreement with a previous study ${ }^{15}$ that shows preferential localization of a mesopropionate-2,6-diiodo-BODIPY in the mitochondria of HSC-2 cells.

\subsection{Singlet oxygen generation studies}

The singlet oxygen quantum yields were determined in DMSO for compounds 5, 7, 8, 10, and 1a-11a by measuring the change in absorbance of singlet oxygen acceptor 1,3diphenylisobenzofuran (DPBF) in the presence of photosensitizer produced singlet oxygen. ${ }^{15,57}$ The change in the $410 \mathrm{~nm}$ absorbance of DPBF (at an initial concentration of $50 \mu \mathrm{M}$ ) was measured in $15 \mathrm{~min}$ intervals over the course of $1 \mathrm{~h}$, and each photosensitizer was referenced to an equivalent concentration of methylene blue. The singlet oxygen quantum yields ranged from 0.02 (for $\mathbf{7 a}$ ) to 0.76 (for 1a), in agreement with literature, ${ }^{58}$ as shown in Table 3. The most phototoxic BODIPYs 2a, 4a and 10a were found to be moderate singlet oxygen generators ( $\Phi_{\Delta}=0.40,0.38$ and 0.34 , respectively) while all other 2,6-diiodo-BODIPYs had $\Phi_{\Delta}<0.32$, with exception of 1a. Although BODIPY 1a has the highest singlet oxygen generation rate of this series of compounds, it was not among the 
four most phototoxic, probably due to its highly lipophilic nature and lack of peripheral functionalization that might favor protein binding. ${ }^{54} \mathrm{On}$ the other hand, BODIPY 7a bearing a meso-thienyl group was the poorest generator of singlet oxygen $\left(\Phi_{\Delta}=0.02\right)$ but still showed high phototoxicity (and low dark toxicity), indicating different mechanism(s) for cell photosensitization.

\section{Conclusions}

A series of eleven photo-stable meso-aryl-BODIPYs, bearing both meso-phenyl and mesothienyl groups, were synthesized and iodinated at the 2,6-positions to investigate the effect of the iodine atoms and the nature of the meso-aryl group on their photophysical properties and cytotoxicity. BODIPYs bearing meso-thienyl and meso-pentafluorophenyl substituents, showed the largest red-shifted absorptions and emissions due to their lower HOMO-LUMO gap, as determined computationally. The 2,6-diiodo-BODIPYs showed lower HOMO and LUMO energies compared with the corresponding non-iodinated derivatives. Furthermore, meso-thienyl BODIPYs showed drastically reduced fluorescence quantum yields due to the greater freedom of rotation of the small thienyl group. Addition of bromine onto the mesosubstitutent had only a slight effect on the rotational barrier and the fluorescence quantum yields.

Studies in human HEp2 cells revealed that all BODIPYs with exception of 10a were nontoxic in the dark $\left(\mathrm{IC}_{50}>400 \mu \mathrm{M}\right)$. Upon light treatment $\left(1.5 \mathrm{~J} / \mathrm{cm}^{2}\right)$ the $\beta$-free BODIPYs showed low cytotoxicity $\left(\mathrm{IC}_{50}>80 \mu \mathrm{M}\right)$ and five of the 2,6-diiodo BODIPYs (3a, 5a, 8a, 9a and 11a) showed no phototoxicity up to $200 \mu \mathrm{M}$. On the other hand, six of the 2,6-diiodoBODIPYs (1a, 2a, 4a, 6a, 7a and 10a) showed $\mathrm{IC}_{50}=3.5-28 \mu \mathrm{M}$ at $1.5 \mathrm{~J} / \mathrm{cm}^{2}$, demonstrating the significant effect of both the 2,6 diiodo and the meso-aryl groups on the cytotoxic properties of BODIPYs. BODIPYs $\mathbf{2 a}$ and $\mathbf{4 a}$ had the highest dark/phototoxicity ratio $(>50)$, and are the most promising for PDT. The high dark and phototoxicity observed for BODIPY 10a are probably due to its very high cellular uptake, and preferential accumulation within the cell mitochondria.

\section{Supplementary Material}

Refer to Web version on PubMed Central for supplementary material.

\section{Acknowledgments}

This work was supported by the National Science Foundation, Grant number CHE-0611629. The authors thank the Louisiana Optical Network Initiative for the use of the computer facilities.

\section{References and notes}

1. Loudet A, Burgess K. Chem Rev. 2007; 107:4891. [PubMed: 17924696]

2. Ulrich G, Ziessel R, Harriman A. Angew Chem, Int Ed. 2008; 47:1184.

3. Benstead M, Mehl GH, Boyle RW. Tetrahedron. 2011; 67:3573.

4. Boens N, Volker Leen V, Dehaen W. Chem Soc Rev. 2012; 41:1130. [PubMed: 21796324]

5. Awuah SG, You Y. RSC Adv. 2012; 2:11169. 
6. Kamkaew A, Lim SH, Lee HB, Kiew LV, Chung LY, Burgess K. Chem Soc Rev. 2013; $42: 77$. [PubMed: 23014776]

7. Dougherty TJ, Gomer CJ, Henderson BW, Jori G, Kessel D, Korbelik M, Moan J, Peng Q. J Nat Cancer Inst. 1998; 90:889. [PubMed: 9637138]

8. Dolmans D, Fukumura D, Jain RK. Nat Rev Cancer. 2003; 3:380. [PubMed: 12724736]

9. Huang Z. Tech Cancer Res Treat. 2005; 4:283.

10. Brown SB, Brown EA, Walker I. Lancet Oncol. 2004; 5:497. [PubMed: 15288239]

11. Jiao L, Li J, Zhang S, Wei C, Hao E, Vicente MGH. New J Chem. 2009; 33:1888.

12. Jiao L, Yu C, Uppal T, Liu M, Li Y, Zhou Y, Hao E, Hu X, Vicente MGH. Org Biomol Chem. 2010; 8:2517. [PubMed: 20390194]

13. Ortiz MJ, Agarrabeitia AR, Duran-Sampedro G, Prieto JB, Lopez TA, Massad WA, Montejano HA, García NA, Arbeloa IL. Tetrahedron. 2012; 68:1153.

14. Yogo T, Urano Y, Ishitsuka Y, Maniwa F, Nagano T. J Am Chem Soc. 2005; 127:12162. [PubMed: 16131160]

15. Lim SH, Thivierge C, Nowak-Sliwinska P, Han J, van den Bergh H, Wagnières G, Burgess K, Lee HB. J Med Chem. 2010; 53:2865. [PubMed: 20199028]

16. Pandey SK, Pan S, Kant R, Kuruvilla SA, Pan ML, Mukherjee J. Bioorg Med Chem Lett. 2012; 22:7610. [PubMed: 23116890]

17. Darwish A, Blacker M, Janzen N, Rathmann SM, Czorny S, Hillier SM, Joyal JL, Babich JW, Valliant JF. ACS Med Chem Lett. 2012; 3:313. [PubMed: 24900470]

18. Tanaka M, Uehara S, Kojima A, Matsumoto M. Phys Med Biol. 2007; 52:4409. [PubMed: 17634641]

19. Agdeppa ED, Spilker ME. AAPS J. 2009; 11:286. [PubMed: 19415506]

20. Lumcke S, Hönnscheidt C, Waschatko G, Bopp A, Lütjohann D, Bertram N, Gehrig-Burger K. Mol Cell Endocrinol. 2010; 314:31. [PubMed: 19755137]

21. Lund FW, Lomholt MA. BMC Biophys. 2012; 5:20. [PubMed: 23078907]

22. Beatty KE, Szychowski J, Fisk JD, Tirrell DA. Chem BioChem. 2011; 12:2137.

23. Atilgan S, Ekmekci Z, Dogan AL, Gucb D, Akkaya EU. Chem Commun. 2006:4398.

24. He H, Lo P-C, Yeung S-L, Fong W-P, Ng DKP. Chem Commun. 2011:4748.

25. Uppal T, Bhupathiraju NVSDK, Vicente MGH. Tetrahedron. 2013; 69:4687.

26. Yang F, Xu XL, Gong YH, Qiu WW, Sun ZR, Zhou JW, Audebert P, Tang J. Tetrahedron. 2007; 63:9188.

27. Kollmannsberger M, Rurack K, Resch-Genger U, Daub J. J Phys Chem A. 1998; 102:10211.

28. Jiao L, Yu C, Li J, Wang Z, Wu M, Hao E. J Org Chem. 2009; 74:7525. [PubMed: 19722510]

29. Ozdemir T, Atilgan S, Kutuk I, Yildirim LT, Tulek A, Bayindir M, Akkaya EU. Org Lett. 2009; 11:2105. [PubMed: 19419209]

30. Wang JG, Hou YJ, Li C, Zhang BW, Wang XS. Sens Actuators, B-Chem. 2011; 157:586.

31. Qin W, Baruah M, De Borggraeve WM, Boens N. J Photochem Photobiol, A: Chem. 2006; 183:190.

32. Zhang XL, Xiao Y, Qian XH. Org Lett. 2008; 10:29. [PubMed: 18069840]

33. Jun T, Kim K, Lee KM, Benniston AC, Churchill DG. J Coord Chem. 2012; 65:4299.

34. Alamiry MAH, Benniston AC, Hagon J, Winstanley TPL, Lemmetyinen H, Tkachenko NV. RSC Adv. 2012; 2:4944.

35. Zhang D, Wang Y, Xiao Y, Qian S, Qian X. Tetrahedron. 2009; 65:8099.

36. Ye JH, Hu ZJ, Wang YX, Zhang WC, Zhang Y. Tetrahedron Lett. 2012; 53:6858.

37. Sabatini RP, McCormick TM, Lazarides T, Wilson KC, Eisenberg R, McCamant DW. J Phys Chem Lett. 2011; 2:223.

38. Mathai S, Smith TA, Ghiggino KP. Photochem Photobiol Sci. 2007; 6:995. [PubMed: 17721599]

39. Didier P, Ulrich G, Mély Y, Ziessel R. Org Biomol Chem. 2009; 7:3639. [PubMed: 19707663]

40. Becke AD. J Chem Phys. 1993; 98:5648.

41. Lee C, Yang W, Parr RG. Phys Rev B. 1988; 37:785. 
42. Stevens WJ, Basch H, Krauss M. J Chem Phys. 1984; 81:6026.

43. Stevens WJ, Krauss M, Basch H, Jasien PG. Can J Chem. 1992; 70:612.

44. Cundari TR, Stevens WJ. J Chem Phys. 1993; 98:5555.

45. Frisch, MJ.; Trucks, GW.; Schlegel, HB.; Robb, MA.; Cheeseman, JR.; Scalmani, G.; Barone, V.; Mennucci, B.; Petersson, GA.; Nakatsuji, H.; Caricato, M.; Li, X.; Hratchian, HP.; Izmaylov, AF.; Bloino, J.; Zheng, G.; Sonnenberg, JL.; Hada, M.; Ehara, M.; Toyota, K.; Fukuda, R.; Hasegawa, J.; Ishida, M.; Nakajima, T.; Honda, Y.; Kitao, O.; Nakai, H.; Vreven, T.; Montgomery, JA.; Peralta, JE.; Ogliaro, F.; Bearpark, M.; Heyd, JJ.; Brothers, E.; Kudin, KN.; Staroverov, VN.; Kobayashi, R.; Normand, J.; Raghavachari, K.; Rendell, N.; Burant, JC.; Iyengar, SS.; Tomasi, J.; Cossi, M.; Rega, N.; Millam, JM.; Klene, N.; Knox, JE.; Cross, JB.; Bakken, V.; Adamo, C.; Jaramillo, J.; Gomperts, R.; Stratmann, RE.; Yazyev, O.; Austin, AJ.; Cammi, R.; Pomelli, C.; Ochterski, JW.; Martin, RL.; Morokuma, K.; Zakrzewski, VG.; Voth, GA.; Salvador, P.; Dannenberg, JJ.; Dapprich, S.; Daniels, AD.; Farkas, O.; Foresman, JB.; Ortiz, JB.; Cioslowski, J.; Fox, DJ. Gaussian, Inc; Wallingford CT: 2009.

46. Meng G, Velayudham S, Smith A, Luck R, Liu HY. Macromolecules. 2009; 42:1995.

47. Mula S, Ray AK, Banerjee M, Chaudhuri T, Dasgupta K, Chattopadhyay S. J Org Chem. 2008; 73:2146. [PubMed: 18302408]

48. Uppal T, Hu X, Fronczek FR, Maschek S, Bobadova-Parvanova P, Vicente MGH. Chem Eur J. 2012; 18:3893. [PubMed: 22367756]

49. Rihn S, Retailleau P, Bugsaliewicz N, De Nicola A, Ziessel R. Tetrahedron Lett. 2009; 50:7008.

50. Fu L, Jiang F-L, Fortin D, Harvey PD, Liu Y. Chem Commun. 2011:5503.

51. Qu XY, Liu Q, Ji XN, Chen HC, Zhou ZK, Shen Z. Chem Commun. 2012:4600.

52. Benniston AC, Copley G. Phys Chem Chem Phys. 2009; 11:4124. [PubMed: 19458813]

53. Kim K, Jo C, Easwaramoorthi S, Sung J, Kim DH, Churchill DG. Inorg Chem. 2010; 49:4881. [PubMed: 20420417]

54. Yogo T, Urano Y, Mizushima A, Sunahara H, Inoue T, Hirose K, Lino M, Kikuchi K, Nagano T. PNAS. 2008; 105:28. [PubMed: 18172220]

55. Weissig V. Exp Opin Drug Delivery. 2005; 2:89.

56. Kessel D, Luo Y. J Photochem Photobiol, B: Biol. 1998; 42:89.

57. Kochevar IE, Redmond RW. Methods Enzymol. 2000; 319:20. [PubMed: 10907495]

58. Huang L, Zhao J, Guo S, Zhang C, Ma J. J Org Chem. 2013; 78:5627. [PubMed: 23668289] 


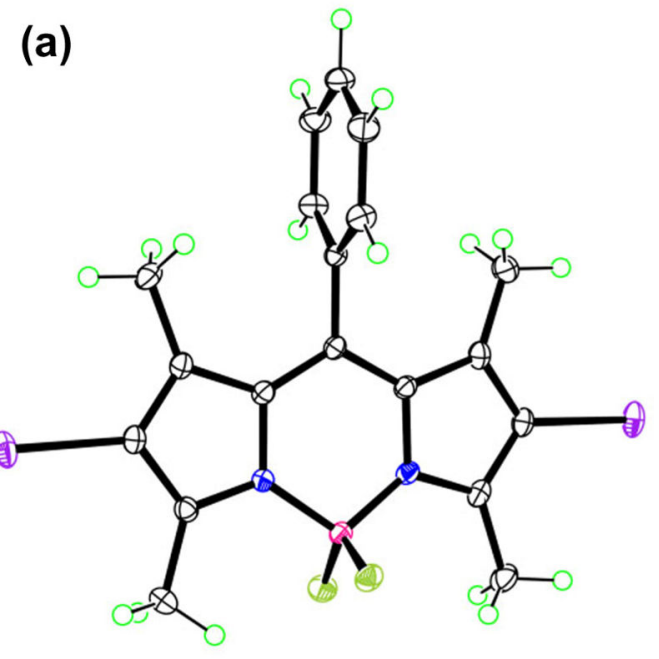

(b)

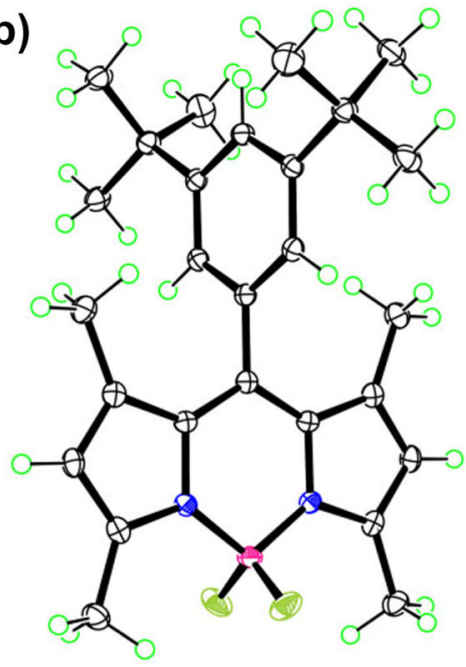

(c)
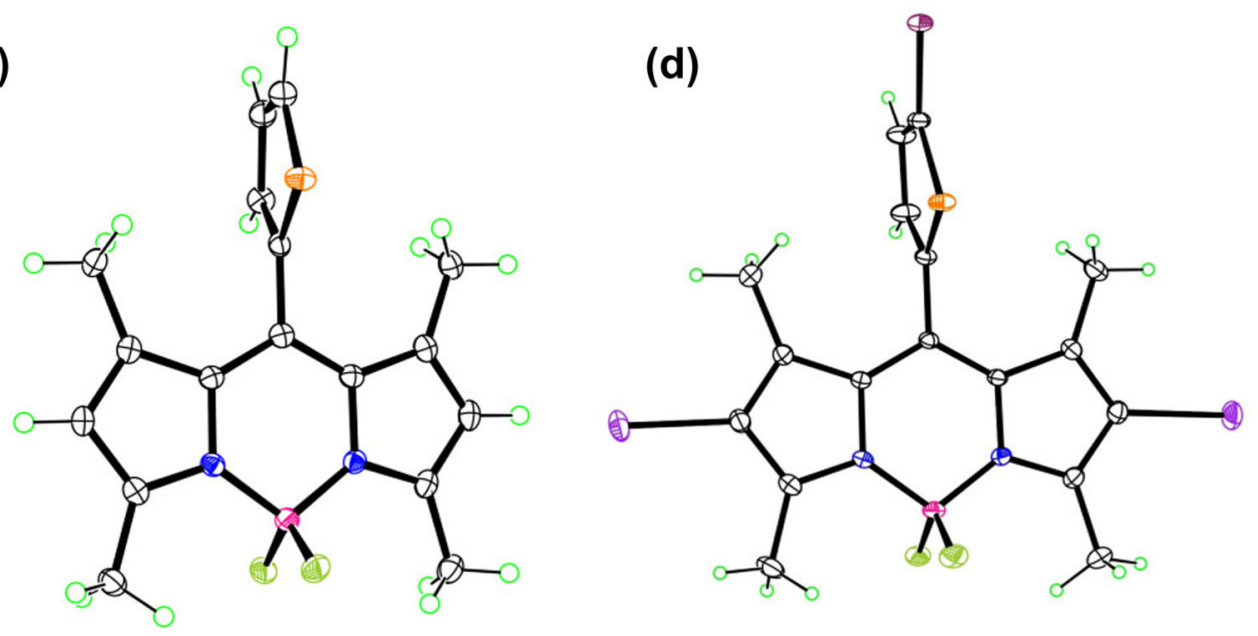

Figure 1.

Molecular structures of BODIPYs 1a (a), 3 (b), 7 (c), and 9a (d) from X-ray crystal structure determinations. Ellipsoids are drawn at the $50 \%$ probability level. 


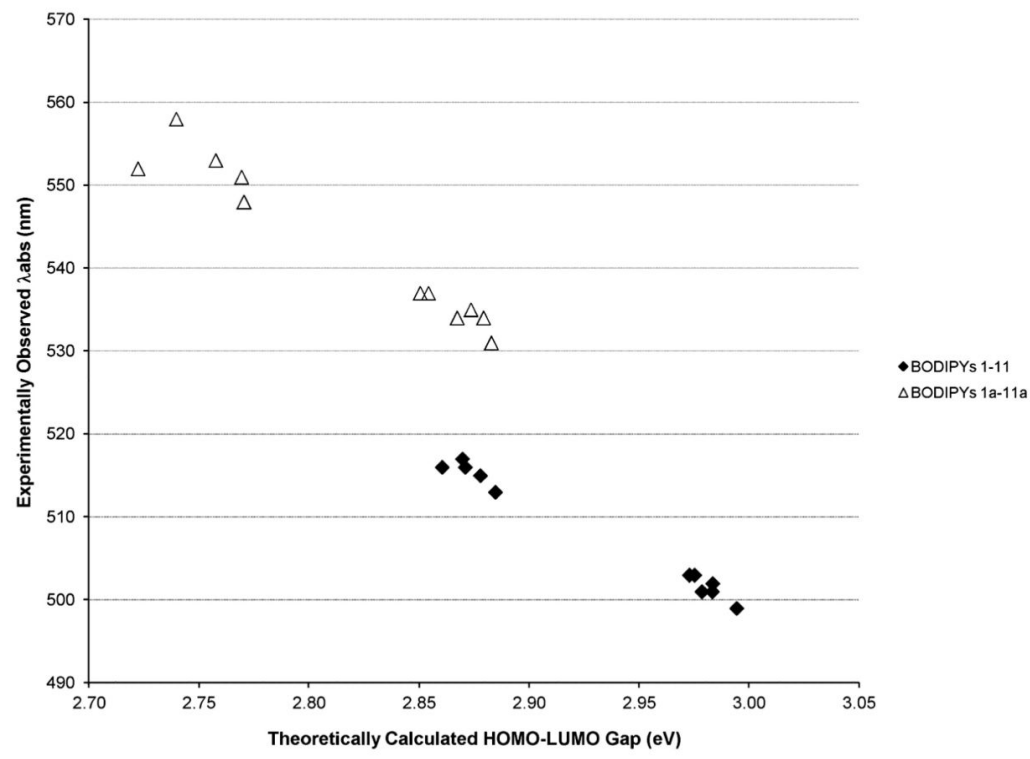

Figure 2.

Experimentally observed absorption wavelengths versus the theoretically calculated HOMO-LUMO gap. 


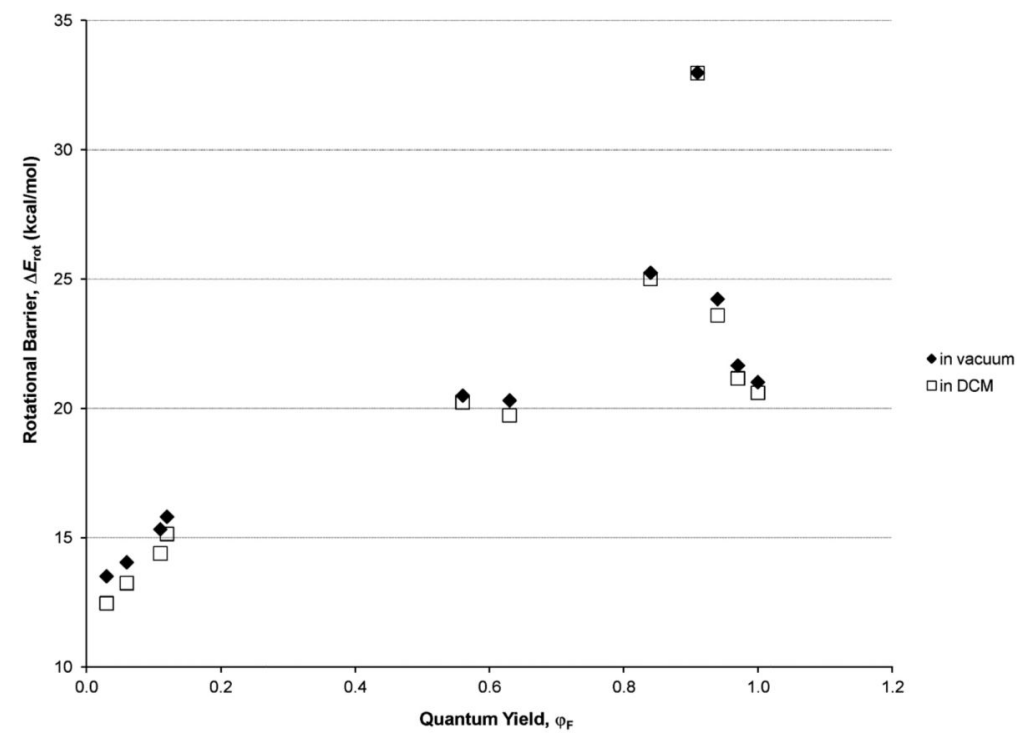

Figure 3.

Theoretically calculated meso-aryl group rotational barrier (in vacuum and in dichloromethane) versus the experimentally observed quantum yield. 


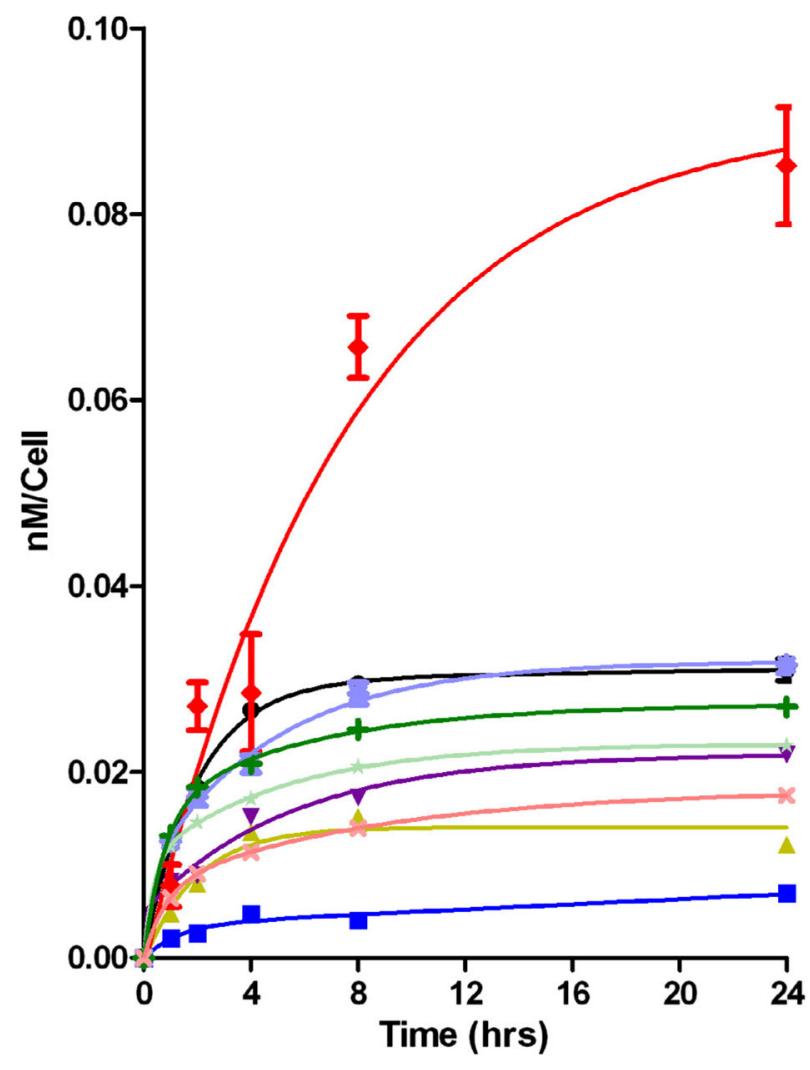

Figure 4.

Time-dependent uptake of meso-aryl-BODIPYs 1a (black), 2a (blue), $4 \mathbf{a}$ (yellow), 5 (light purple), 6a (purple), 7 (light green), 8 (dark green), 10 (pink) and 10a (red) at $10 \mu \mathrm{M}$ by human HEp2 cells. 

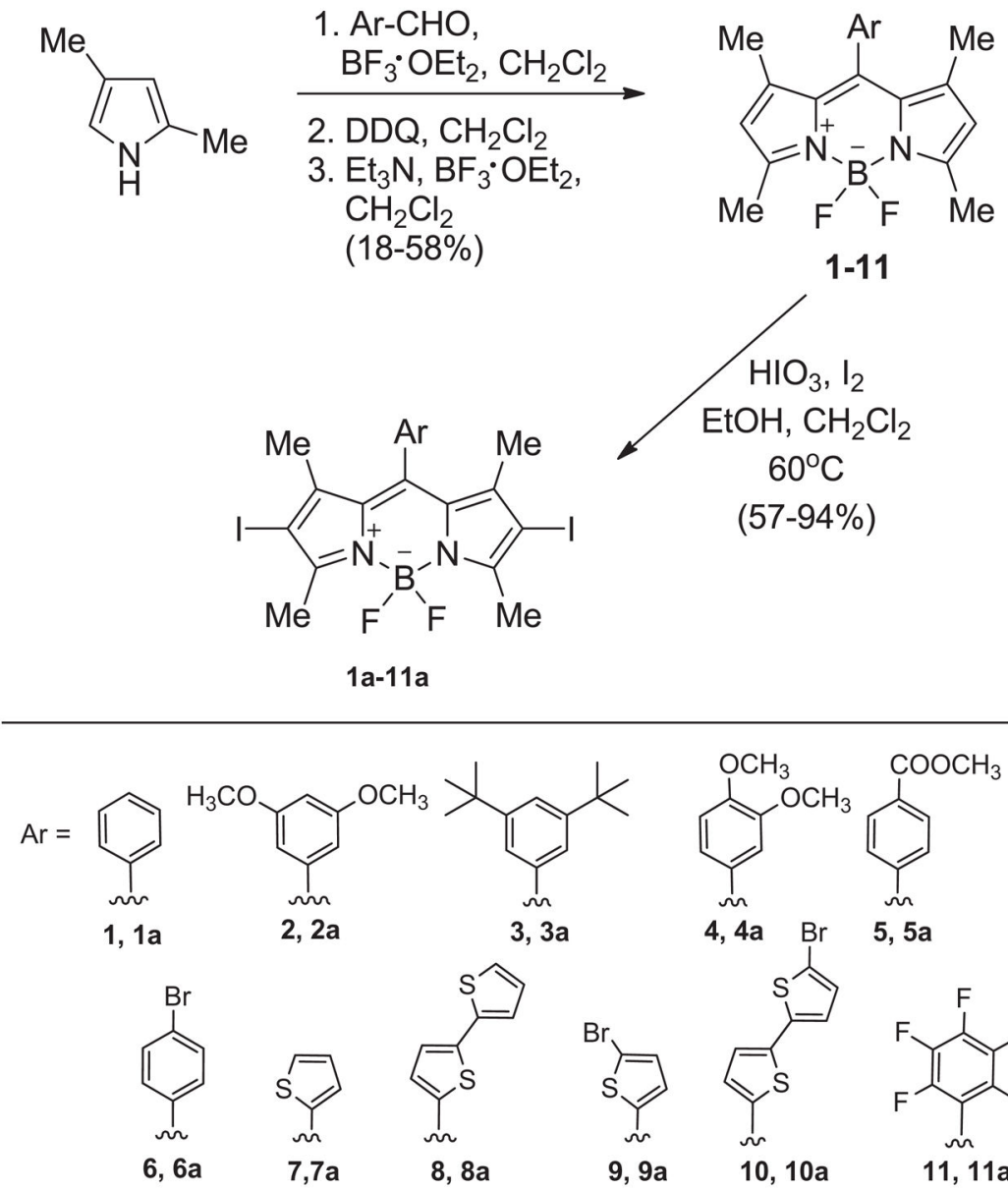

4, 4a $\mathrm{Br} \quad 5,5 \mathrm{a}$
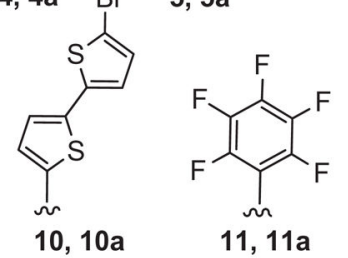

Scheme 1. 


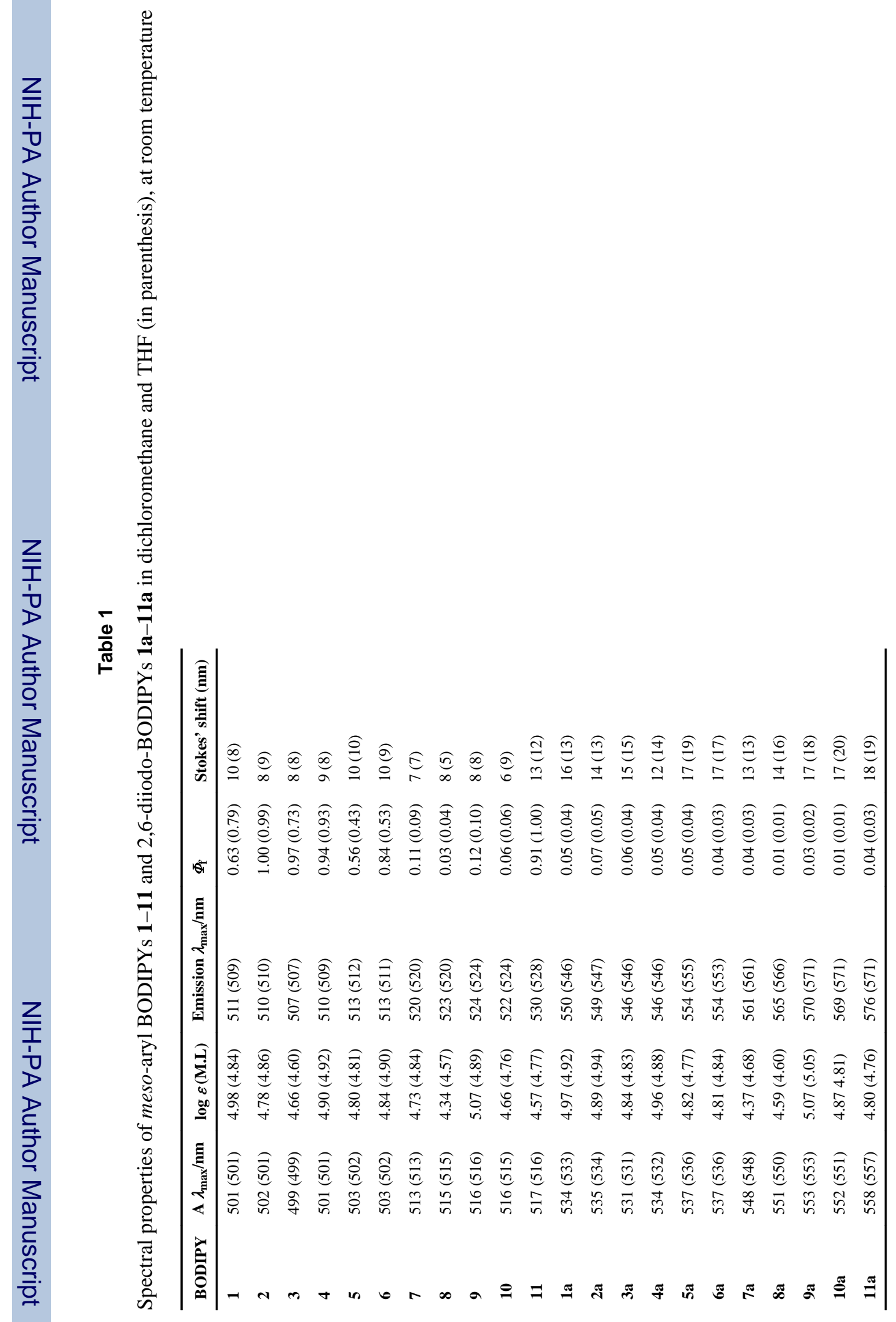




\section{Table 2}

Theoretically calculated energies of HOMO (a.u.), LUMO (a.u.), HOMO-LUMO gap, $E_{\mathrm{g}}(\mathrm{eV})$, and mesogroup rotational barrier, $\Delta E_{\mathrm{rot}}(\mathrm{kcal} / \mathrm{mol})$ in vacuum and in dichloromethane (in parentheses), for BODIPYs 1-

11a

\begin{tabular}{lllll}
\hline BODIPY & HOMO & LUMO & $\boldsymbol{E}_{\mathbf{g}}$ & $\Delta \boldsymbol{E}_{\text {rot }}$ \\
\hline $\mathbf{1}$ & -0.2072 & -0.0976 & 2.98 & $20.3(19.7)$ \\
$\mathbf{2}$ & -0.2028 & -0.0931 & 2.98 & $21.0(20.6)$ \\
$\mathbf{3}$ & -0.2048 & -0.0948 & 2.99 & $21.7(21.2)$ \\
$\mathbf{4}$ & -0.2068 & -0.0973 & 2.98 & $24.2(18.6)$ \\
$\mathbf{5}$ & -0.2107 & -0.1013 & 2.98 & $20.5(20.2)$ \\
$\mathbf{6}$ & -0.2112 & -0.1020 & 2.97 & $25.3(25.0)$ \\
$\mathbf{7}$ & -0.2090 & -0.1030 & 2.88 & $15.3(14.4)$ \\
$\mathbf{8}$ & -0.2093 & -0.1035 & 2.88 & $13.6(12.5)$ \\
$\mathbf{9}$ & -0.2124 & -0.1073 & 2.86 & $15.8(15.2)$ \\
$\mathbf{1 0}$ & -0.2113 & -0.1058 & 2.87 & $14.1(13.3)$ \\
$\mathbf{1 1}$ & -0.2189 & -0.1134 & 2.87 & $33.0(33.0)$ \\
$\mathbf{1 a}$ & -0.2159 & -0.1106 & 2.87 & - \\
$\mathbf{2 a}$ & -0.2117 & -0.1061 & 2.87 & - \\
$\mathbf{3 a}$ & -0.2136 & -0.1076 & 2.88 & - \\
$\mathbf{4 a}$ & -0.2152 & -0.1094 & 2.88 & - \\
$\mathbf{5 a}$ & -0.2188 & -0.1139 & 2.85 & - \\
$\mathbf{6 a}$ & -0.2195 & -0.1147 & 2.85 & - \\
$\mathbf{7 a}$ & -0.2175 & -0.1157 & 2.77 & - \\
$\mathbf{8 a}$ & -0.2173 & -0.1155 & 2.77 & - \\
$\mathbf{9 a}$ & -0.2204 & -0.1190 & 2.76 & - \\
$\mathbf{1 0 a}$ & -0.2222 & -0.1222 & 2.72 & - \\
$\mathbf{1 1 a}$ & -0.2260 & -0.1253 & 2.74 & - \\
\hline & & & & \\
\hline
\end{tabular}




\section{Table 3}

Comparative singlet oxygen quantum yields (relative to methylene blue), dark and phototoxicity (at $1.5 \mathrm{~J} / \mathrm{cm}^{2}$ light dose) of selected BODIPYs toward HEp2 cells using the Cell Titer Blue assay

\begin{tabular}{lllll}
\hline BODIPY & Dark toxicity $\mathbf{I C}_{\mathbf{5 0}}(\boldsymbol{\mu M})$ & Phototoxicity $_{\mathbf{I C}} \mathbf{C H}_{\mathbf{5 0}}(\boldsymbol{\mu M})$ & Ratio & $\Phi_{\boldsymbol{\Delta}}$ \\
\hline $\mathbf{5}$ & $>400$ & $>100$ & $>4$ & 0.18 \\
$\mathbf{7}$ & $>300$ & $>100$ & $>3$ & 0.10 \\
$\mathbf{8}$ & $>400$ & $>100$ & $>4$ & 0.29 \\
$\mathbf{1 0}$ & $>300$ & 82 & $>3.5$ & 0.38 \\
$\mathbf{1 a}$ & $>400$ & 27 & $>15$ & 0.76 \\
$\mathbf{2 a}$ & $>400$ & 4.0 & $>100$ & 0.40 \\
$\mathbf{3 a}$ & $>400$ & $>200$ & $>2$ & 0.32 \\
$\mathbf{4 a}$ & $>400$ & 7.5 & $>53$ & 0.38 \\
$\mathbf{5 a}$ & $>400$ & $>200$ & $>2$ & 0.27 \\
$\mathbf{6 a}$ & $>400$ & 28 & $>15$ & 0.31 \\
$\mathbf{7 a}$ & $>400$ & 14 & $>30$ & 0.02 \\
$\mathbf{8 a}$ & $>400$ & $>200$ & $>2$ & 0.27 \\
$\mathbf{9 a}$ & $>400$ & $>200$ & $>2$ & 0.10 \\
$\mathbf{1 0 a}$ & 8 & 3.5 & 2.3 & 0.34 \\
$\mathbf{1 1 a}$ & $>400$ & $>200$ & $>2$ & 0.19 \\
\hline
\end{tabular}

